\title{
TEXAS
}

WATER

DEVELOPMENT

$B O A R D$

Report 197

\section{GROUND-WATER DATA FOR ORANGE COUNTY AND VICINITY, TEXAS AND LOUISIANA, 1971-74}



REPORT 197

\section{GROUND-WATER DATA FOR ORANGE COUNTY \\ AND VICINITY, TEXAS AND LOUISIANA, 1971-74}

By

C. W. Bonnet

United States Geological Survey

\footnotetext{
This report was prepared by the U.S. Geological Survey under cooperative agreement with the Texas Water Development Board, the Sabine River Authority of Texas; and the Orange County Commissioner's Court
} 


\section{TEXAS WATER DEVELOPMENT BOARD}

John H. McCoy, Chairman

W. E. Tinsley

Carl Illig
Robert B. Gilmore, Vice Chairman Milton Potts

A. L. Black

Harry P. Burleigh, Executive Director

Authorization for use or reproduction of any original material contained in this publication, i.e., not obtained from other sources, is freely granted. The Board would appreciate acknowledgement.

Published and distributed

by the

Texas Water Development Board

Post Office Box 13087

Austin, Texas 78711 
Purpose and Scope . . . . . . . . . . . . . . . . . . . . . . . . . . . . .

Well-Numbering System . . . . . . . . . . . . . . . . . . . . . . . . . . . . . . .

GROUND.WATER DATA . . . . . . . . . . . . . . . . . . . . . . . . . . . . . . .

Water-Level Measurements . . . . . . . . . . . . . . . . . . . . . . 1

Chemical Analyses of Water From Wells . . . . . . . . . . . . . . . . . . . . . . 2

Ground-Water Withdrawals . . . . . . . . . . . . . . . . . . . . . . . . . 2

SELECTED REFERENCES . . . . . . . . . . . . . . . . . . . . . . . . . . . . 5

\section{TABLES}

1. Ground-Water Withdrawals in Orange County, 1963-74 . . . . . . . . . . . . . . . .

2. Water-Level Measurements in the Orange County Area, 1971-74 . . . . . . . . . . . . . 6

3. Chemical Analyses of Water From Wells in the Orange County Area, 1971-74 . . . . . . . . . - 20

\section{FIGURES}

1. Hydrographs Showing Changes in Water Levels in Wells UJ-62-59-105 and CU-530 . . . . . . . 3

2. Map Showing Locations of Water-Level Observation Wells . . . . . . . . . . . . . . . . . 25

3. Map Showing Locations of Chemical-Quality Observation Wells . . . . . . . . . . . . . 26 
1 


\section{GROUND.WATER DATA FOR ORANGE COUNTY \\ AND VICINITY, TEXAS AND LOUISIANA, 1971-74}

\section{INTRODUCTION}

\section{Purpose and Scope}

The program of continuing ground-water studies in Orange County and adjacent areas began in March 1967 because of the need for a systematic program to monitor the changing ground-water conditions. The data-collection program, conducted by the U.S. Geological Survey in cooperation with the Texas Water Development Board, the Sabine River Authority of Texas, and the Orange County Commissioner's Court, consists of water-level measurements in observation wells, the collection of water samples from wells for chemical analyses, an inventory of new large-capacity wells, and pumping tests on new large-capacity wells.

This report presents the data collected during the period September 1971 to October 1974 on water-level measurements, chemical analyses of water from welis, and ground-water withdrawals.

The English units used in this report may be converted to metric units by the following conversion factors:

\begin{tabular}{lcl}
\multicolumn{1}{c}{ FROM } & MLLTIPLY BY & TO OBTAIN \\
feet & 0.3048 & meters \\
$\begin{array}{l}\text { million gallons } \\
\text { per day }\end{array}$ & 3.785 & $\begin{array}{c}\text { million liters } \\
\text { per day }\end{array}$
\end{tabular}

\section{Well-Numbering System}

The well-numbering system in Texas was developed by the Texas Water Development Board for use throughout the State. Under this system, each 1-degree quadrangle is given a number consisting of two digits. These are the first two digits in the well number. Each 1-degree quadrangle is divided into $7 \frac{1}{2}$-minute quadrangles which are given two-digit numbers from 01 to 64. These are the third and fourth digits of the well number. Each 7/2-minute quadrangle is divided into $21 / 2$-minute quadrangles which are given a single-digit number from 1 to 9 . This is the fifth digit of the well number. Finally, each well within a $2 \frac{1}{2}-$ minute quadrangle is given a two-digit number in the order in which it was inventoried, starting with 01 . These are the last two digits of the well number.

Only the last three digits of the well number are shown at each well location (Figure 2); the second two digits are shown in the northwest corner of each $7 \frac{1}{2-m i n u t e}$ quadrangle; and the first two digits are shown by the large block numerals in each 1-degree quadrangle.

In addition to the seven-digit well number, a two-fetter prefix is used to identify the county. The prefixes for Orange and adjacent counties are as follows: Orange, UJ; Jasper, PR; Jefferson, PT; Hardin, LH; and Newton, TZ.

Wells inventoried in Louisiana by the Geological Survey are assigned a number consisting of two parts, an abbreviation of the name of the parish in which the well is located and a serial number that designates the well. The number assigned does not indicate a specific location because the number is generally assigned in the order in which the well was inventoried. The prefix for Calcasieu Parish is CU; the prefix for Cameron Parish is CN.

\section{GROUND-WATER DATA}

\section{Water-Level Measurements}

A network of 116 observation wells was maintained for water-level measurements from 1971 to 1974. The area within the network includes Orange County, the southern parts of Jasper and Newton Counties, the eastern part of Jefferson County, the southeastern part of Hardin County, all in Texas; and the western parts of Cameron and Calcasieu Parishes, Louisiana. The heaviest concentration of observation. 

wells is within the industrial area in the southern part of Orange County. The locations of water-level observation wells are shown on Figure 2.

Static water-level measurements were made generally in the early part of the year at the time of least interference from pumping and maximum recovery from the previous heavy seasonal pumping. Pumping water-level measurements were made during the latter part of the year at the time of maximum drawdown of water levels. These data are needed to study the relationships between water levels and withdrawals at the time of maximum effects. In addition to the periodically measured wells, one well has been selected for continuous monitoring of water levels. All static and pumping water-level measurements and selected measurements from the continuous monitor are given in Table 2. Hydrographs of two wells with the longest periods of record are shown on Figure 1 .

\section{Chemical Analyses of Water From Wells}

A network of 76 wells to observe changes in chemical quality of the ground water was maintained from 1971 to 1974 in Orange County. Water samples were obtained from most of these wells during the latter part of each year. During these periods, the maximum concentrations of chlorides are expected.

Locations of the chemical-quality observation wells are shown on Figure 3 . The results of chemical analyses are given in Table 3.

\section{Ground-Water Withdrawals}

Data on ground-water pumpage for all major uses during the period 1963.74 are given in Table 1. These data are essential to any study of the cause and effect relationships between pumping and the decline in water levels or changes in chemical quality. 


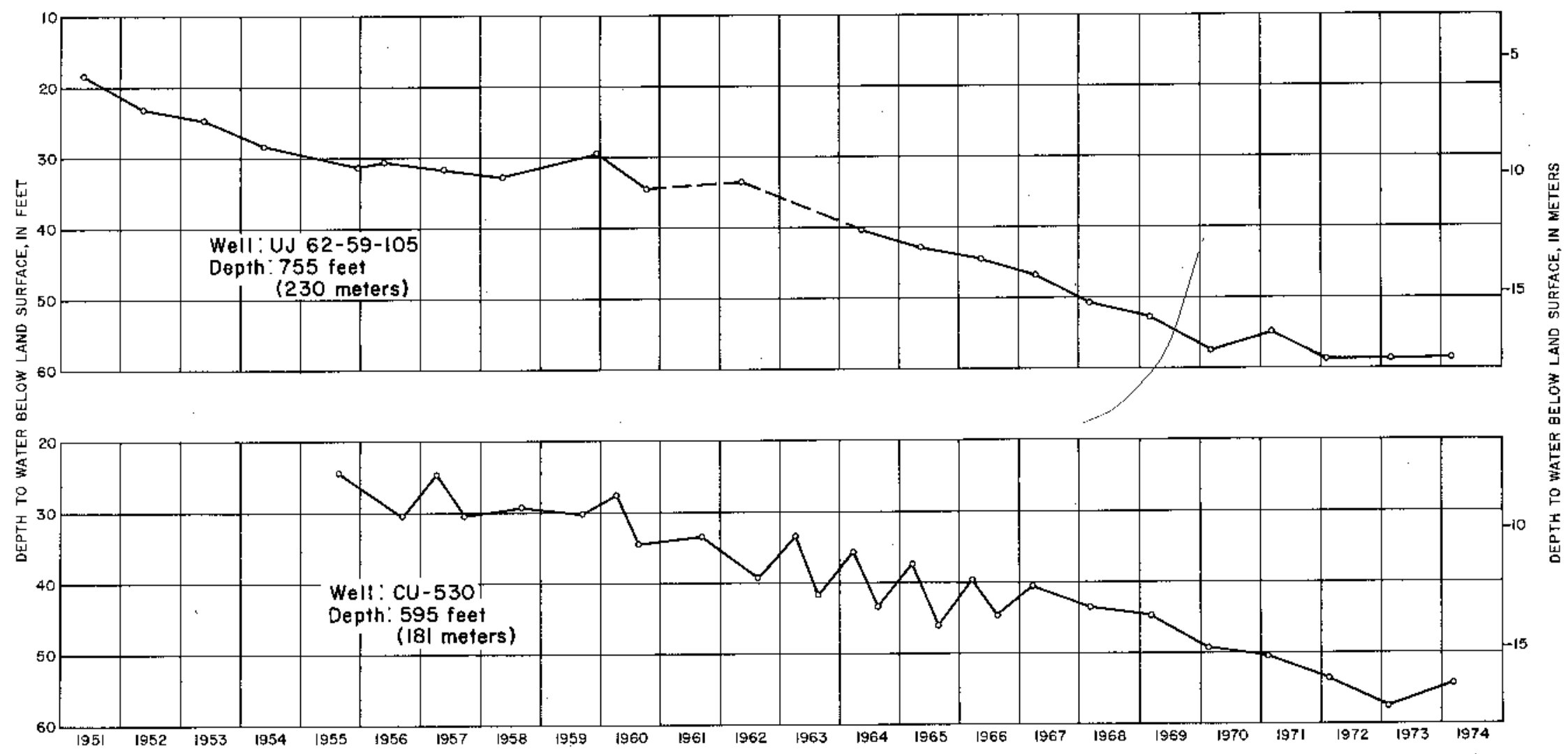

Figure 1

Changes in Water Levels in Wells

UJ-62-59-105 and CU-530 
Table 1.-Ground-water withdrawals in Orange County, 1963-74.

(million gallons per day)

$\begin{array}{lccc}\text { YEAR } & \begin{array}{c}\text { MUNICIPAL } \\ \text { SUPPLY }\end{array} & \begin{array}{c}\text { INDUSTRIAL } \\ \text { USE }\end{array} & \text { TOTAL } \\ 1963 & 3.8 & 14.7 & 18.5 \\ 1964 & 4.1 & 16.2 & 20.3 \\ 1965 & 4.5 & 16.3 & 20.8 \\ 1966 & 4.7 & 16.3 & 21.0 \\ 1967 & 5.8 & 14.7 & 20.5 \\ 1968 & 4.6 & 16.6 & 21.2 \\ 1969 & 4.8 & 16.0 & 20.8 \\ 1970 & 5.1 & 15.9 & 21.0 \\ 1971 & 5.4 & 17.6 & 23.0 \\ 1972 & 5.4 & 17.7 & 23.1 \\ 1973 & 5.3 & 15.8 & 21.1 \\ 1974 & 5.5 & 15.0 & 20.5\end{array}$




\section{SELECTED REFERENCES}

Gabrysch, R. K., and McAdoo, G. D., 1972, Development of ground-water resources in the Orange County area, Texas and Louisiana, 1963-71: Texas Water Devel. Board Rept. 156, 47 p., 10 figs.

McAdoo, G. D., 1968, Ground-water data for Orange County and vicinity, Texas and Louisiana, 1968: U.S. Geol. Survey open-file rept., $20 \mathrm{p}$.

1969. Ground-water data for Orange County and vicinity, Texas and Louisiana, 1969: U.S. Geol. Survey open-file rept., $22 \mathrm{p}$.
McAdoo, G. D., 1970, Ground-water data for Orange County and vicinity, Texas and Louisiana, 1970: U.S. Geol. Survey open-file rept., 19 p.

Wesselman, J. B., 1965, Geology and ground-water resources of Orange County. Texas: Texas Water Comm. Bull. 6516, $112 \mathrm{p}$. 
$x$ 
Table 2.--Water-level measurements in the Orange County area, 1971-74

\begin{tabular}{|c|c|c|c|c|c|c|}
\hline $\begin{array}{l}\text { We11 } \\
\text { no. }\end{array}$ & Owner & $\begin{array}{c}\text { Screened } \\
\text { interval } \\
\text { or } \\
\text { depth } \\
\text { (feet) } \\
\end{array}$ & $\begin{array}{l}\text { Date } \\
\text { of } \\
\text { measurement }\end{array}$ & $\begin{array}{l}\text { Static } \\
\text { water-level } \\
\text { below land } \\
\text { surface datum } \\
\text { (feet) }\end{array}$ & $\begin{array}{c}\text { Date } \\
\text { of } \\
\text { measurement }\end{array}$ & $\begin{array}{c}\text { Pumping } \\
\text { water-level } \\
\text { below land } \\
\text { surface datum } \\
\text { (feet) }\end{array}$ \\
\hline $\begin{array}{l}\text { Orange County } \\
\text { UJ-61-56-103 }\end{array}$ & B. H. Thibodeau & 76 & $\begin{array}{l}2-7-72 \\
3-19-73 \\
3-18-74\end{array}$ & $\begin{array}{r}14.47 \\
12.79 \\
9.28\end{array}$ & $\begin{array}{l}-- \\
-- \\
--\end{array}$ & $\begin{array}{l}-- \\
-- \\
--\end{array}$ \\
\hline $61-56-116$ & H. H. Houseman & 800 & $\begin{array}{l}2-7-72 \\
3-19-73 \\
3-18-74\end{array}$ & $\begin{array}{l}37.95 \\
37.59 \\
36.52\end{array}$ & $\begin{array}{l}-- \\
-- \\
--\end{array}$ & $\begin{array}{l}-- \\
-- \\
--\end{array}$ \\
\hline $61-56-314$ & L. B. Wi11iamson & $375-385$ & $\begin{array}{l}6-29-72 \\
3-19-73 \\
3-18=74\end{array}$ & $\begin{array}{l}39.68 \\
38.82 \\
37.87\end{array}$ & $\begin{array}{l}-- \\
--\end{array}$ & $\begin{array}{l}-- \\
-- \\
--\end{array}$ \\
\hline $61-56-315$ & Iwanda Trailer Park & $356-380$ & $\begin{array}{l}6-29-72 \\
3-19-73 \\
3-18-74\end{array}$ & $\begin{array}{l}39.69 \\
38.80 \\
37.62\end{array}$ & $\begin{array}{l}-- \\
-- \\
--\end{array}$ & $\begin{array}{l}-- \\
--\end{array}$ \\
\hline $61-56-611$ & Bढ̧B Water System & $441-457$ & $\begin{array}{l}2-7-72 \\
3-19-73 \\
3-18-74\end{array}$ & $\begin{array}{l}40.65 \\
41.46 \\
38.14\end{array}$ & $\begin{array}{l}-- \\
-- \\
--\end{array}$ & $\begin{array}{l}-- \\
-- \\
-\end{array}$ \\
\hline $61-56-901$ & Orange County WCGID No. 1 , wel1 2 & $350-400$ & $\begin{array}{c}-- \\
2-11-72 \\
3-23-73 \\
3-22-74\end{array}$ & $\begin{array}{l}-- \\
45.20 \\
42.45 \\
40.00\end{array}$ & $\begin{array}{r}9-16-71 \\
9-27-72 \\
9-25-73 \\
10-17-74\end{array}$ & $\begin{array}{l}75.23 \\
80.62 \\
82.76 \\
86.27\end{array}$ \\
\hline $61-56-911$ & B\&B Water System & $468-486$ & $\begin{array}{l}2-7-72 \\
3-19-73 \\
3-18-74\end{array}$ & $\begin{array}{l}37.76 \\
38.77 \\
38.38\end{array}$ & $\begin{array}{l}-- \\
-- \\
--\end{array}$ & $\begin{array}{l}-- \\
-- \\
--\end{array}$ \\
\hline $6 I-56-919$ & Orange County WCEID No. 1 , we11 3 & $385-420$ & $\begin{array}{c}-1-72 \\
2-11-72 \\
3-23-73 \\
3-22-74\end{array}$ & $\begin{array}{l}\ldots \\
51.15 \\
52.60 \\
50.54\end{array}$ & $\begin{array}{r}9-16-71 \\
9-27-72 \\
9-25-73 \\
10-17-74\end{array}$ & $\begin{array}{l}61.07 \\
62.89 \\
63.38 \\
68.00\end{array}$ \\
\hline
\end{tabular}


Table 2.--Water-level measurements in the Orange County area, 1971-74--Continued

\begin{tabular}{|c|c|c|c|c|c|c|c|}
\hline $\begin{array}{l}\text { Well } \\
\text { no. }\end{array}$ & Owner & & $\begin{array}{l}\text { Screened } \\
\text { interval } \\
\text { or } \\
\text { depth } \\
\text { (feet) } \\
\end{array}$ & $\begin{array}{l}\text { Date } \\
\text { of } \\
\text { measurement }\end{array}$ & $\begin{array}{c}\text { Static } \\
\text { water-level } \\
\text { below land } \\
\text { surface datum } \\
\text { (feet) } \\
\end{array}$ & $\begin{array}{c}\text { Date } \\
\text { of } \\
\text { measurement }\end{array}$ & $\begin{array}{c}\text { Pumping } \\
\text { water-level } \\
\text { below land } \\
\text { surface datum } \\
\text { (feet) } \\
\end{array}$ \\
\hline$U J-61-56-920$ & B\&्ष B Water System & & 380 & $\begin{array}{l}2-7-72 \\
3-19-73 \\
3-18-74\end{array}$ & $\begin{array}{l}43.4 \\
44.35 \\
44.10\end{array}$ & $\begin{array}{l}- \\
-- \\
--\end{array}$ & $\begin{array}{l}-- \\
-- \\
--\end{array}$ \\
\hline $62-49-503$ & G. L. Linscomb & & 117 & $\begin{array}{l}2-7-72 \\
3-26-73 \\
3-26-74\end{array}$ & $\begin{array}{r}12.73 \\
8.88 \\
8.54\end{array}$ & $\begin{array}{l}-- \\
-- \\
--\end{array}$ & $\begin{array}{l}-- \\
-- \\
--\end{array}$ \\
\hline $62-49-904$ & Texas Highway Department & & $399-415$ & $\begin{array}{l}2-7-72 \\
3-19-73 \\
3-18-74\end{array}$ & $\begin{array}{l}41.70 \\
42.98 \\
42.45\end{array}$ & $\begin{array}{l}-- \\
-- \\
--\end{array}$ & $\begin{array}{l}-- \\
-- \\
--\end{array}$ \\
\hline $62-50-201$ & Boyce Ward & & $476-586$ & $\begin{array}{l}2-9-72 \\
3-27-73 \\
3-20-74\end{array}$ & $\begin{array}{l}44.72 \\
44.73 \\
44.08\end{array}$ & $\begin{array}{l}-- \\
-- \\
--\end{array}$ & $\begin{array}{l}-- \\
-- \\
--\end{array}$ \\
\hline $62-50-602$ & Huber Oi1 Co. & & $380-400$ & $\begin{array}{c}2-9-72 \\
3-22-73 \\
\text { Measurements }\end{array}$ & $\begin{array}{c}45.61 \\
46.67 \\
\text { discontinued }\end{array}$ & -- & $\begin{array}{l}-- \\
--\end{array}$ \\
\hline $62-50-807$ & Frank Michell & & $442-454$ & $\begin{array}{l}6-30-72 \\
3-20-73 \\
3-21-74\end{array}$ & $\begin{array}{l}50.70 \\
49.52 \\
49.04\end{array}$ & $\begin{array}{l}-- \\
-- \\
--\end{array}$ & $\begin{array}{l}-- \\
-- \\
--\end{array}$ \\
\hline $62-50-808$ & H. D. Womack & $\ldots$ & $643-655$ & $3-20-74$ & 51.99 & - & -- \\
\hline $62-50-904$ & George G1idden & & 566 & $\begin{array}{l}2-7-72 \\
3-28-73 \\
3-20-74\end{array}$ & $\begin{array}{r}10.89 \\
8.59 \\
5.98\end{array}$ & $\begin{array}{l}-- \\
-- \\
--\end{array}$ & $\begin{array}{l}-- \\
-- \\
--\end{array}$ \\
\hline $62-51-103$ & Owens I11inois, Inc. & & $445-515$ & $\begin{array}{l}6-28-72 \\
3-27-73 \\
3-20-74\end{array}$ & $\begin{array}{r}49.38 \\
44.86 \\
46.04\end{array}$ & $\begin{array}{l}-- \\
-- \\
--\end{array}$ & $\begin{array}{l}-- \\
-- \\
--\end{array}$ \\
\hline
\end{tabular}



TabIe 2.--Water-leveI measurements in the Orange County area, 1971-74--Contfnued

\begin{tabular}{|c|c|c|c|c|c|c|}
\hline $\begin{array}{l}\text { Well } \\
\text { no. }\end{array}$ & Owner & $\begin{array}{l}\text { Screened } \\
\text { interval } \\
\text { or } \\
\text { depth } \\
\text { (feet) } \\
\end{array}$ & $\begin{array}{c}\text { Date } \\
\text { of } \\
\text { measurement }\end{array}$ & $\begin{array}{c}\text { Static } \\
\text { water-level } \\
\text { below land } \\
\text { surface datum } \\
\text { (feet) }\end{array}$ & $\begin{array}{c}\text { Date } \\
\text { of } \\
\text { measurement }\end{array}$ & $\begin{array}{c}\text { Pumping } \\
\text { water-level } \\
\text { below land } \\
\text { surface datum } \\
\text { (feet) } \\
\end{array}$ \\
\hline $\mathrm{UJ}-62-51-104$ & Owens Illinois, Inc. & $460-470$ & $\begin{array}{l}6-28-72 \\
3-27-73 \\
3-20-74\end{array}$ & $\begin{array}{l}52.02 \\
47.80 \\
48.70\end{array}$ & $\begin{array}{l}-- \\
-- \\
--\end{array}$ & $\begin{array}{l}-- \\
-- \\
--\end{array}$ \\
\hline $\begin{array}{c}62-51-706 \\
.\end{array}$ & Phillips Chemical Co. & $428-488$ & $\begin{array}{l}-- \\
-- \\
--\end{array}$ & $\begin{array}{l}-- \\
-- \\
-- \\
--\end{array}$ & $\begin{array}{r}9-20-71 \\
9-21-72 \\
10-2-73 \\
10-22-74\end{array}$ & $\begin{array}{l}67.28 \\
66.41 \\
66.10 \\
66.22\end{array}$ \\
\hline $62-51-707$ & $\begin{array}{l}\text { do. } \\
\quad \\
\quad-\end{array}$ & $428+488$ & $\begin{array}{l}2-9-72 \\
3-27-73 \\
3-28-74\end{array}$ & $\begin{array}{l}50.49 \\
50.51 \\
52.18\end{array}$ & $\begin{array}{l}-- \\
-- \\
--\end{array}$ & -- \\
\hline $62-57-203$ & K. Kishi & 740 & $\begin{array}{l}2+11-72 \\
3-20-73 \\
3-21-74\end{array}$ & $\begin{array}{l}46.13 \\
46.92 \\
46.99\end{array}$ & $\begin{array}{ll}-- \\
-- \\
--\end{array}$ & $\begin{array}{ll}-- \\
--\end{array}$ \\
\hline $62-57-401$ & Texas Eastem Transmission Co. & $448-468$ & $\begin{array}{l}2+11-72 \\
3-23-73 \\
3-21-74\end{array}$ & $\begin{array}{l}45.57 \\
46.27 \\
46.72\end{array}$ & $\begin{array}{l}-- \\
--\end{array}$ & $\begin{array}{l}-- \\
-- \\
--\end{array}$ \\
\hline $62-57-403$ & Gulf States Utilities Co., Vidor, well 1 & $433-483$ & $3-\overline{22-74}$ & --.14 & $\begin{array}{r}9-27-73 \\
10-23-74\end{array}$ & $\begin{array}{l}68.75 \\
69.85\end{array}$ \\
\hline $62-57-404$ & Gulf States Utilities Co., Vidor, weIl 2 & $430-481$ & $\begin{array}{l}3-23-73 \\
3-21-74\end{array}$ & $\begin{array}{l}32.32 \\
30.11\end{array}$ & -- & $\begin{array}{l}- \\
-\end{array}$ \\
\hline $62-57-405$ & Gulf States Utilities Co., Vidor, well 3 & $430-480$ & $\begin{array}{l}2-9-72 \\
3-23-73 \\
3-22-74\end{array}$ & $\begin{array}{l}36.54 \\
34.94 \\
38.84\end{array}$ & $\begin{array}{c}-- \\
-- \\
10-23-74\end{array}$ & $\begin{array}{l}-- \\
-- \\
89.29\end{array}$ \\
\hline $62-57-406$ & Gulf States Utilities Co, , Vidor, weil 6 & $430-480$ & $\begin{array}{l}3-23-73 \\
3-21-74\end{array}$ & $\begin{array}{l}42.17 \\
45.88\end{array}$ & $10-\overline{-22-74}$ & $8 \overrightarrow{1.88}$ \\
\hline
\end{tabular}


i 
Table 2.--Water-level measurements in the Orange County area, 1971-74--Continued

\begin{tabular}{|c|c|c|c|c|c|c|}
\hline $\begin{array}{l}\text { Wel1 } \\
\text { no. }\end{array}$ & Owner & $\begin{array}{l}\text { Screened } \\
\text { interval } \\
\text { or } \\
\text { depth } \\
\text { (feet) } \\
\end{array}$ & $\begin{array}{l}\text { Date } \\
\text { of } \\
\text { measurement }\end{array}$ & $\begin{array}{c}\text { Static } \\
\text { water-level } \\
\text { below land } \\
\text { surface datum } \\
\text { (feet) }\end{array}$ & $\begin{array}{l}\text { Date } \\
\text { of } \\
\text { measurement }\end{array}$ & $\begin{array}{c}\text { Pumping } \\
\text { watcr-level } \\
\text { below land } \\
\text { surfacer datum } \\
\text { (fedt) }\end{array}$ \\
\hline UJ $-62-57-407$ & Gulf States Utilities Co., Vidor, well 4 & $320-370$ & $3-22-74$ & 37.80 & $10-23-74$ & 59.54 \\
\hline $62-57-408$ & Gulf States Utilities Co, Vidor, well 5 & $343-383$ & $3-22-74$ & 40.72 & $10-23-74$ & 86.12 \\
\hline $62-57-409$ & L. N. Michael & $550-640$ & $\begin{array}{l}2-11-72 \\
3-23-73 \\
3-21-74\end{array}$ & $\begin{array}{l}43.91 \\
44.72 \\
44.98\end{array}$ & $\begin{array}{l}-- \\
-- \\
--\end{array}$ & $\begin{array}{ll}-- \\
-- \\
--\end{array}$ \\
\hline $62-57-501$ & Florida Gas Co. & $405-435$ & $\begin{array}{l}2-11-72 \\
3-23-73 \\
3-21-74\end{array}$ & $\begin{array}{l}43.69 \\
44.03 \\
44.64\end{array}$ & $\begin{array}{l}-- \\
-- \\
--\end{array}$ & $\begin{array}{l}-- \\
-- \\
--\end{array}$ \\
\hline $62-57-904$ & Gulf States Utilities Co., Sabine, well 4 & $432-455$ & $\begin{array}{c}1-24-72 \\
3-27-73 \\
--\end{array}$ & $\begin{array}{l}89.5^{*} \\
93.5^{*} \\
--\end{array}$ & $\begin{array}{l}8-72 \\
10-1-73 \\
10-23-74\end{array}$ & $\begin{array}{l}155.5^{*} \\
155.5^{*} \\
152.5^{*}\end{array}$ \\
\hline $62-57-905$ & Gulf States Utilities Co., Sabine, well 5 & $422-461$ & $\begin{array}{l}1-24-72 \\
3-27-73\end{array}$ & $\begin{array}{l}84.5^{*} \\
90.5^{*}\end{array}$ & $\begin{array}{l}-- \\
--\end{array}$ & -- \\
\hline $62-57-907$ & Gulf States Utilities Co., Sabine, well 7 & $604-654$ & $\begin{array}{l}1-24-72 \\
3-23-73 \\
3-28-74\end{array}$ & $\begin{array}{l}39.5 * \\
40.5 * \\
43.37\end{array}$ & $\begin{array}{l}8-72 \\
-- \\
--\end{array}$ & $\begin{array}{l}76.5^{*} \\
-- \\
--\end{array}$ \\
\hline $62-57-908$ & Gu1f States Utilities Co,, Sabine, well 8 & $573-623$ & $3-28-74$ & 42.33 & $6-72$ & $70^{*}$ \\
\hline $62-58-304$ & Orange County WCEID No, 2 , well 1 & $626-706$ & $\begin{array}{l}2-9-72 \\
3-20-73 \\
3-19-74\end{array}$ & $\begin{array}{l}-- \\
62.57 \\
59.11 \\
59.54\end{array}$ & $\begin{array}{r}9-16-71 \\
9-20-72 \\
9-26-73 \\
10-16-74\end{array}$ & $\begin{array}{l}72.78 \\
76.08 \\
72.79 \\
68.46\end{array}$ \\
\hline * Water level & eported. & . & & & & \\
\hline
\end{tabular}



Table 2.--Water-1eve1 measurements in the Orange County area, 1971-74--Continued

\begin{tabular}{|c|c|c|c|c|c|c|}
\hline $\begin{array}{c}\text { Well } \\
\text { no. }\end{array}$ & Owner & $\begin{array}{l}\text { Screened } \\
\text { interva } 1 \\
\text { or } \\
\text { depth } \\
\text { (feet) } \\
\end{array}$ & $\begin{array}{c}\text { Date } \\
\text { of } \\
\text { measurement }\end{array}$ & $\begin{array}{c}\text { Static } \\
\text { water-1evel } \\
\text { below land } \\
\text { surface datum } \\
\text { (feet) }\end{array}$ & $\begin{array}{c}\text { Date } \\
\text { of } \\
\text { measurement }\end{array}$ & $\begin{array}{c}\text { Pumping } \\
\text { water-level } \\
\text { below land } \\
\text { surface datum } \\
\text { (foet) }\end{array}$ \\
\hline$U J-62-58-305$ & City of Orange, Pinehurst well & $520-610$ & $\begin{array}{l}2-8-72 \\
3-20-73 \\
3-19-74\end{array}$ & $\begin{array}{l}60.47 \\
53.75 \\
53.90\end{array}$ & $\begin{array}{r}9-19-72 \\
9-24.73 \\
10-16-74\end{array}$ & $\begin{array}{l}95.20 \\
93.57 \\
93.75\end{array}$ \\
\hline $62-58-324$ & City of Pinehurst & $365-445$ & $\begin{array}{c}-- \\
2-9-72 \\
3-20-73 \\
3-19-74\end{array}$ & $\begin{array}{l}-- \\
59.04 \\
55.15 \\
54.41\end{array}$ & $\begin{array}{r}9-16-71 \\
9-20-72 \\
10-2-73 \\
10-16-74\end{array}$ & $\begin{array}{l}108.70 \\
110.95 \\
109.69 \\
108.10\end{array}$ \\
\hline $62-58-325$ & Orange County WC\&ID No. 2 , welI 2 & $620-670$ & $\begin{array}{c}-- \\
2-9-72 \\
3-20-73 \\
3-19-74\end{array}$ & $\begin{array}{l}-- \\
61.13 \\
59.15 \\
58.75\end{array}$ & $\begin{array}{r}9-16-71 \\
9-20-72 \\
9-26-73 \\
10-16-74\end{array}$ & $\begin{array}{l}78.54 \\
81.03 \\
76.58 \\
75.26\end{array}$ \\
\hline $62-58-403$ & Orangefield Ind. Schoo1 District & $460-480$ & $\begin{array}{l}2-7-72 \\
3-20-73 \\
3-21-74\end{array}$ & $\begin{array}{l}35.40 \\
37.62 \\
38.93\end{array}$ & $\begin{array}{l}-- \\
-- \\
--\end{array}$ & $\begin{array}{l}-- \\
-- \\
--\end{array}$ \\
\hline $62-58-410$ & Orangefield Recreation Park & $110-120$ & $\begin{array}{l}2-7-72 \\
3-20-73 \\
3-21-74\end{array}$ & $\begin{array}{l}6.01 \\
4.62 \\
4.07\end{array}$ & $\begin{array}{l}-- \\
-- \\
--\end{array}$ & $\begin{array}{l}-- \\
-- \\
--\end{array}$ \\
\hline $62-58-602$ & Donnar Corp. & 711 & $\begin{array}{l}2-8-72 \\
3-28-73 \\
3-19-74\end{array}$ & $\begin{array}{l}62.28 \\
60.83 \\
60.69\end{array}$ & $\begin{array}{l}-- \\
-- \\
--\end{array}$ & $\begin{array}{l}-- \\
-- \\
--\end{array}$ \\
\hline $62-58-603$ & W. H. Stark Estate & 204 & $\begin{array}{l}2-7-72 \\
3-19-74\end{array}$ & $\begin{array}{l}8.68 \\
8.19\end{array}$ & $\begin{array}{l}-- \\
--\end{array}$ & $m$ \\
\hline $62-58-605$ & Gulf Chemical Co., wel1 4 & $604-717$ & $3-20-74$ & 61.44 & $9-17-71$ & 74.14 \\
\hline $62-58-606$ & Gulf Chemical Co., well 3 & $630-710$ & -- & -- & $9-21-72$ & 71.00 \\
\hline
\end{tabular}


Table 2.--Water-level measurements in the Orange County area, 1971-74--Continued

\begin{tabular}{|c|c|c|c|c|c|c|}
\hline $\begin{array}{l}\text { Well } \\
\text { no. }\end{array}$ & . & $\begin{array}{l}\text { Screencd } \\
\text { interval } \\
\text { or } \\
\text { depth } \\
\text { (feet) } \\
\end{array}$ & $\begin{array}{c}\text { Date } \\
\text { of } \\
\text { measurement }\end{array}$ & $\begin{array}{l}\text { Static } \\
\text { water-level } \\
\text { below land } \\
\text { surface datum } \\
\text { (feet) }\end{array}$ & $\begin{array}{c}\text { Date } \\
\text { of } \\
\text { measurement }\end{array}$ & $\begin{array}{l}\text { Pumping } \\
\text { water-1evel } \\
\text { below land } \\
\text { surface datum } \\
\text { (feet) }\end{array}$ \\
\hline UJ $-62-58-608$ & Allied Chemical Co. & $620-735$ & $\begin{array}{l}-- \\
-- \\
-- \\
--\end{array}$ & $\begin{array}{l}-- \\
-- \\
-- \\
--\end{array}$ & $\begin{array}{r}9-17-71 \\
9-20-72 \\
9-26-73 \\
10-17-74\end{array}$ & $\begin{array}{l}81.13 \\
84.87 \\
86.11 \\
67.98\end{array}$ \\
\hline $62-58-609$ & E. I. DuPont Co., we11 103-3 & $634-723$ & $\begin{array}{l}-- \\
-- \\
-- \\
--\end{array}$ & $\begin{array}{l}-- \\
-- \\
-- \\
--\end{array}$ & $\begin{array}{r}9-21-71 \\
9-20-72 \\
9-26-73 \\
10-18-74\end{array}$ & $\begin{array}{r}100.46 \\
101.52 \\
104.04 \\
99.93\end{array}$ \\
\hline $62-58-610$ & E. I. DuPont Co., well 103-3,1 & 715 & $\begin{array}{l}-- \\
-- \\
--\end{array}$ & $\begin{array}{l}-- \\
-- \\
--\end{array}$ & $\begin{array}{l}8-8-72 \\
3-21-73 \\
3-28-74\end{array}$ & $\begin{array}{l}64.36 \\
62.67 \\
62.74\end{array}$ \\
\hline $62-58-611$ & E. I. DuPont Co., well 103-2 & 715 & $\begin{array}{l}3-20-73 \\
3-28-74\end{array}$ & $\begin{array}{l}61.53 \\
61.16\end{array}$ & $\begin{array}{l}-- \\
--\end{array}$ & -- \\
\hline $62-58-612$ & E. I. DuPont Co., we11 103-4 & 735 & $\begin{array}{l}2-8-72 \\
3-21-73 \\
3-28-74\end{array}$ & $\begin{array}{l}62.05 \\
60.46 \\
60.80\end{array}$ & $\begin{array}{l}-- \\
-- \\
--\end{array}$ & $\begin{array}{l}-- \\
-- \\
--\end{array}$ \\
\hline $62-58-613$ & E. I. DuPont Co., well 103-1.1 & 723 & $\begin{array}{l}2-8-72 \\
3-21-73 \\
3-28-74\end{array}$ & $\begin{array}{l}63.40 \\
61.99 \\
61.09\end{array}$ & $\begin{array}{l}-- \\
-- \\
--\end{array}$ & $\begin{array}{l}-- \\
-- \\
--\end{array}$ \\
\hline $62-58-614$ & E. I. DuPont Co., we11 103-I & 726 & $\begin{array}{l}-- \\
-- \\
-- \\
--\end{array}$ & $\begin{array}{l}-- \\
-- \\
-- \\
--\end{array}$ & $\begin{array}{r}9-21-71 \\
9-20-72 \\
9-26-73 \\
10-18-74\end{array}$ & $\begin{array}{l}77.69 \\
89.82 \\
87.80 \\
81.85\end{array}$ \\
\hline
\end{tabular}




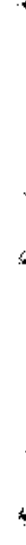


Table 2.--Water-leve1 measurements in the Orange County area, 1971-74--Continued

\begin{tabular}{|c|c|c|c|c|c|c|}
\hline $\begin{array}{c}\text { We11 } \\
\text { no. }\end{array}$ & Owner & $\begin{array}{l}\text { Screened } \\
\text { interva1 } \\
\text { or } \\
\text { depth } \\
\text { (feet) } \\
\end{array}$ & $\begin{array}{c}\text { Date } \\
\text { of } \\
\text { measurement }\end{array}$ & $\begin{array}{c}\text { Static } \\
\text { water-leve1 } \\
\text { below land } \\
\text { surface daturn } \\
\text { (feet) }\end{array}$ & $\begin{array}{c}\text { Date } \\
\text { of } \\
\text { measurement }\end{array}$ & $\begin{array}{c}\text { Pumping } \\
\text { water-leve1 } \\
\text { below land } \\
\text { surface datum } \\
\text { (feet) } \\
\end{array}$ \\
\hline UJ $-62-58-615$ & Firestone Petrochemical Center, well $\mathrm{P}-817$ & 700 & $\begin{array}{c}-- \\
-- \\
3-21-73 \\
3-20-74\end{array}$ & $\begin{array}{c}-- \\
-- \\
55.96 \\
55.80\end{array}$ & $\begin{array}{c}9-17-71 \\
9-22-72 \\
-- \\
--\end{array}$ & $\begin{array}{l}74 \\
70.72 \\
-- \\
--\end{array}$ \\
\hline $62-58-616$ & Gulf Chemical Co., wel1 2 & 718 & $\begin{array}{l}2-8-72 \\
3-21-73 \\
3-20-74\end{array}$ & $\begin{array}{l}61.55 \\
59.06 \\
58.94\end{array}$ & $\begin{array}{l}-- \\
-- \\
--\end{array}$ & $\begin{array}{l}-- \\
-- \\
--\end{array}$ \\
\hline $62-58-618$ & E. I. DuPont Co., well 103-6 & $637-682$ & $\begin{array}{l}2-8-72 \\
3-21-73 \\
3-28-74\end{array}$ & $\begin{array}{l}56.55 \\
55.27 \\
54.89\end{array}$ & $\begin{array}{l}-- \\
-- \\
--\end{array}$ & -- \\
\hline $62-58-629$ & Firestone Petrochemica1 Center, well P-821 & $595-680$ & - & $\begin{array}{l}-- \\
--\end{array}$ & $\begin{array}{r}9-17-71 \\
10-1-73\end{array}$ & $\begin{array}{l}57.05 \\
80\end{array}$ \\
\hline $62-58-631$ & Firestone Petrocherica1 Center, we11 P-826 & $585-680$ & $\begin{array}{l}2-8-72 \\
3-21-73 \\
3-20-74\end{array}$ & $\begin{array}{l}59.38 \\
57.47 \\
57.48\end{array}$ & $\begin{array}{l}-- \\
-- \\
--\end{array}$ & $\begin{array}{l}-- \\
-- \\
--\end{array}$ \\
\hline $62-58-632$ & B. F. Goodrich Co., well 1 & $640-710$ & $\begin{array}{c}-- \\
-- \\
3-21-73 \\
--\end{array}$ & $\begin{array}{l}-- \\
-- \\
55.72 \\
--\end{array}$ & $\begin{array}{r}9-17-71 \\
9-21-72 \\
9-26-73 \\
10-22-74\end{array}$ & $\begin{array}{l}70.30 \\
70.17 \\
74.27 \\
67.62\end{array}$ \\
\hline $62-58-633$ & B. F. Goodrich Co., we11 2 & $625-725$ & $\begin{array}{l}2-8-72 \\
3-29-74\end{array}$ & $\begin{array}{l}58.79 \\
55.99\end{array}$ & +- & -- \\
\hline $62-58-634$ & B. F. Goodrich Co., well 3 & $614-715$ & $\begin{array}{l}-- \\
-- \\
3-21-73 \\
--\end{array}$ & $\begin{array}{l}-- \\
-- \\
58.04 \\
--\end{array}$ & $\begin{array}{r}9-17-71 \\
9-21-72 \\
9-26-73 \\
10-22-74\end{array}$ & $\begin{array}{l}72.10 \\
70.96 \\
73.37 \\
61.74\end{array}$ \\
\hline
\end{tabular}




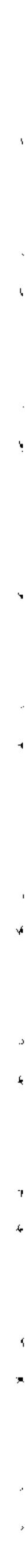


Table 2, water-1evel measurements in the Orange County area, 1971-74--Cont1nued

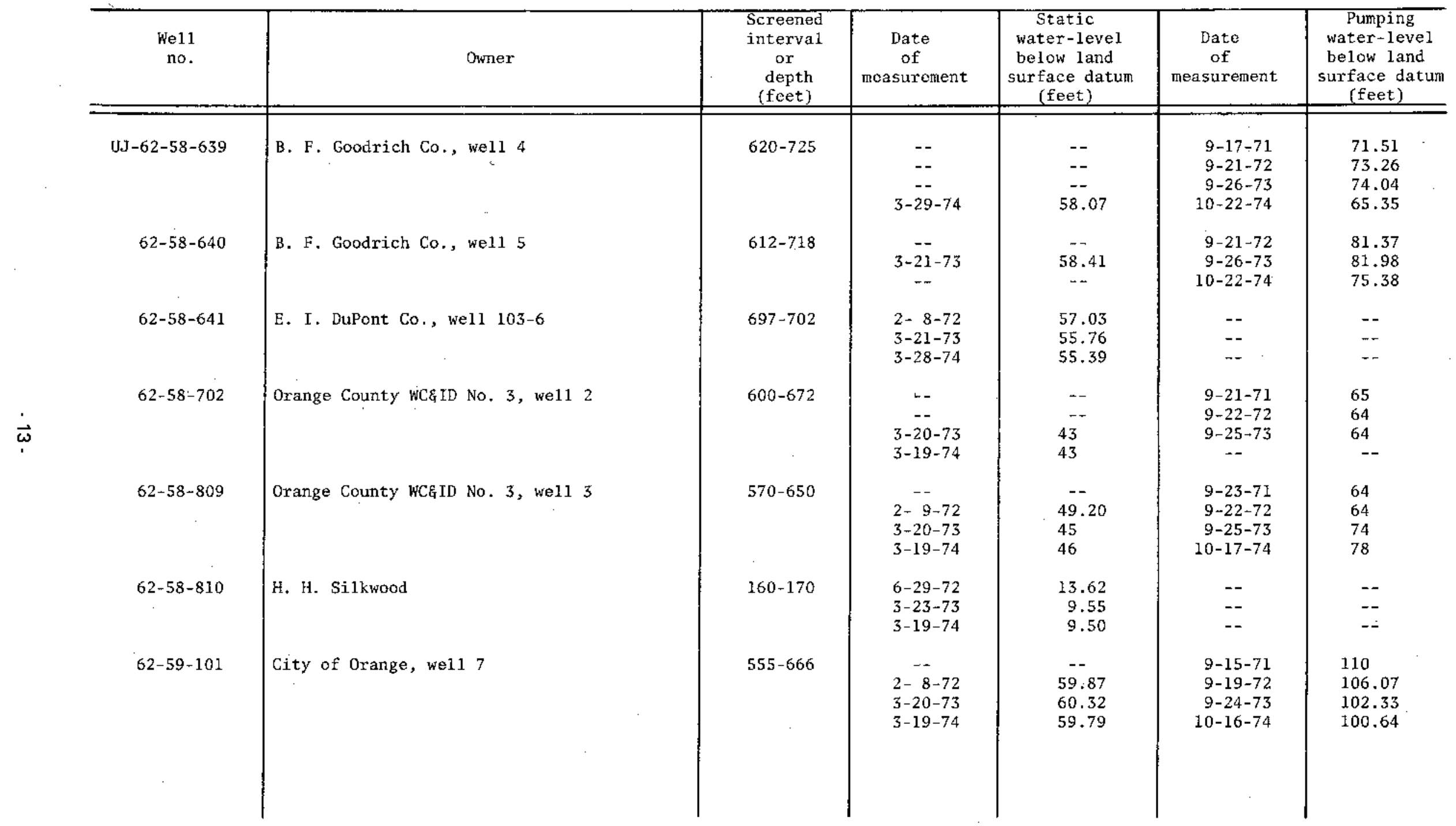


Table 2.--Water-level measurements in the Orange County area, 1971-74--Continued

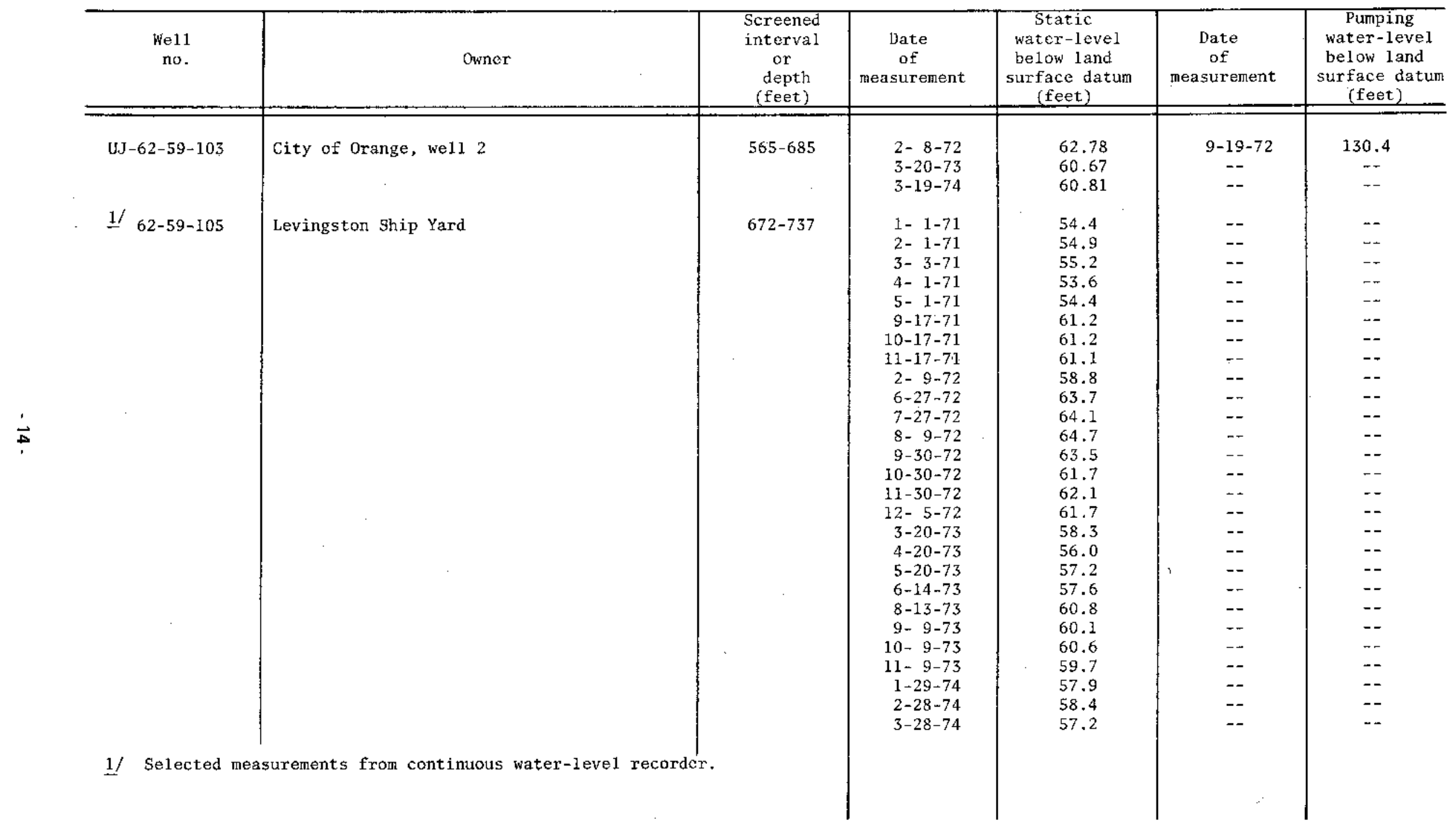




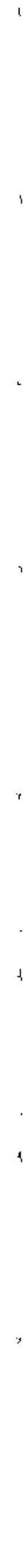


Table 2.--Water-level measurements in the Orange County area, 1971-74--Continued

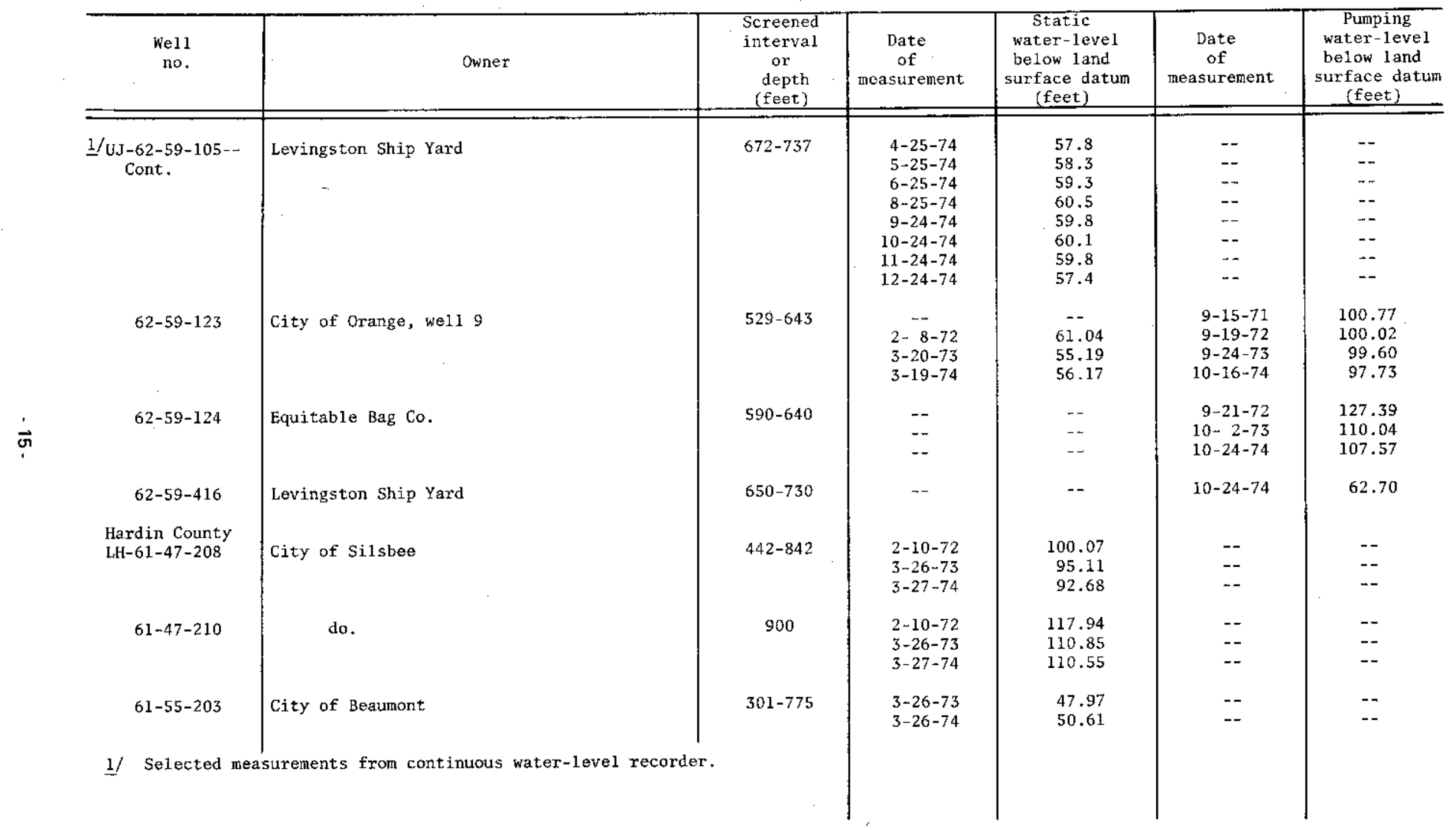


Table 2.--Water-1eve1 measurements in the Orange County area, 1971-74--Continued

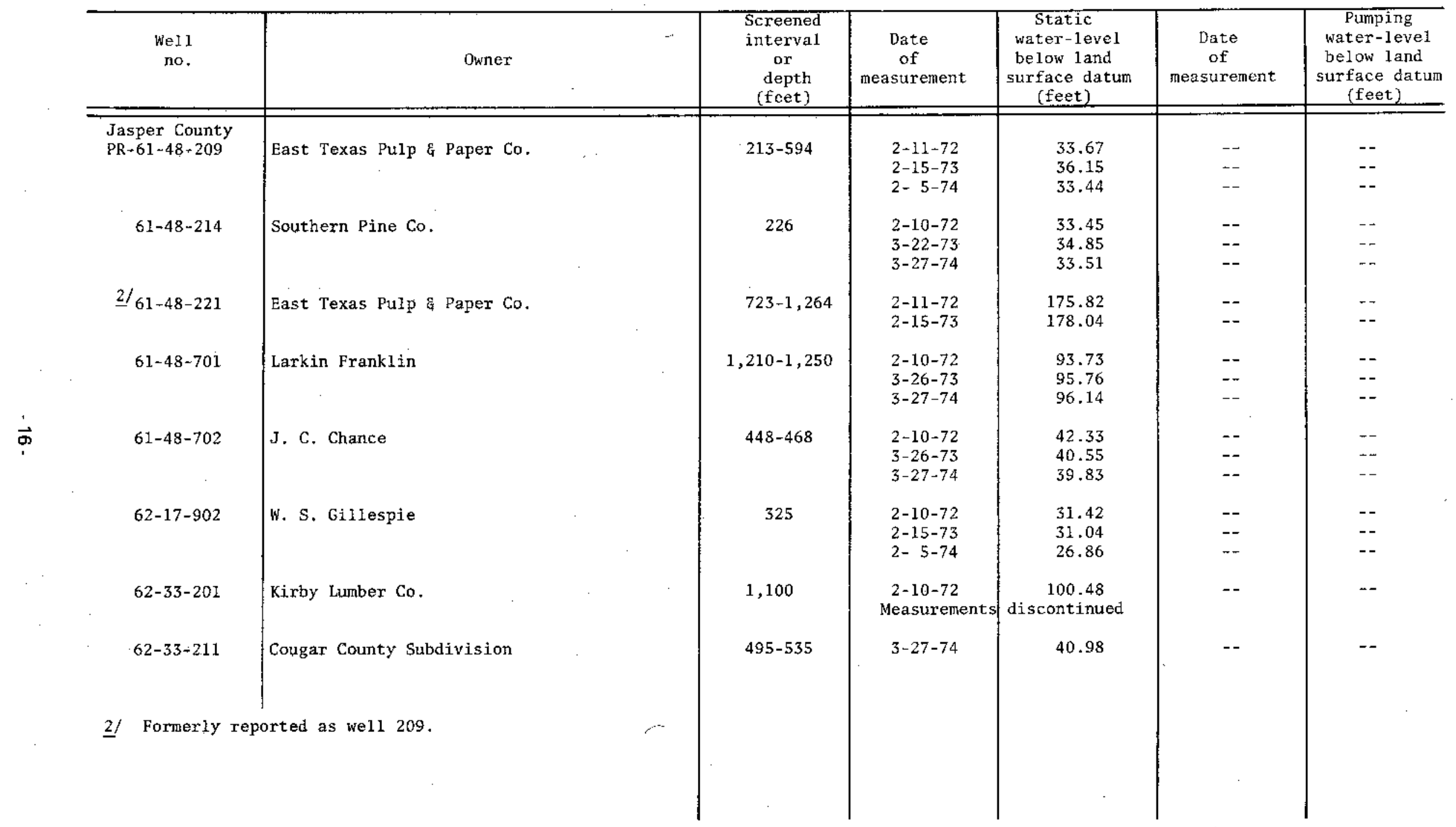



Table 2.--Water-level measurements in the Orange County area, 1971-74--Continued

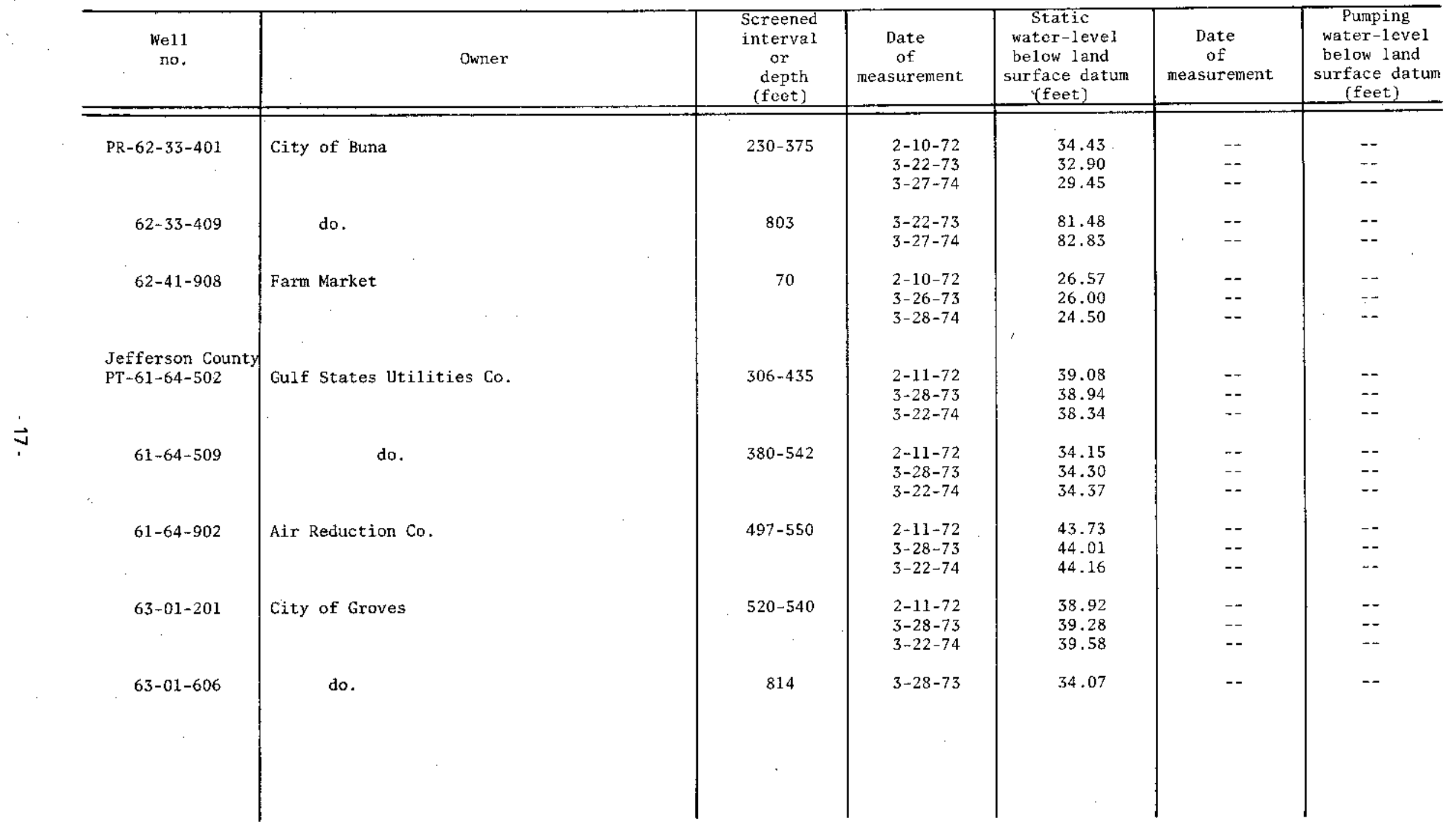




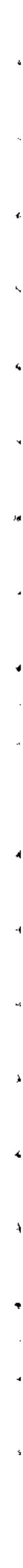


Table 2.--Water-leve1 measurements in the Orange County area, 1971-74--Continued

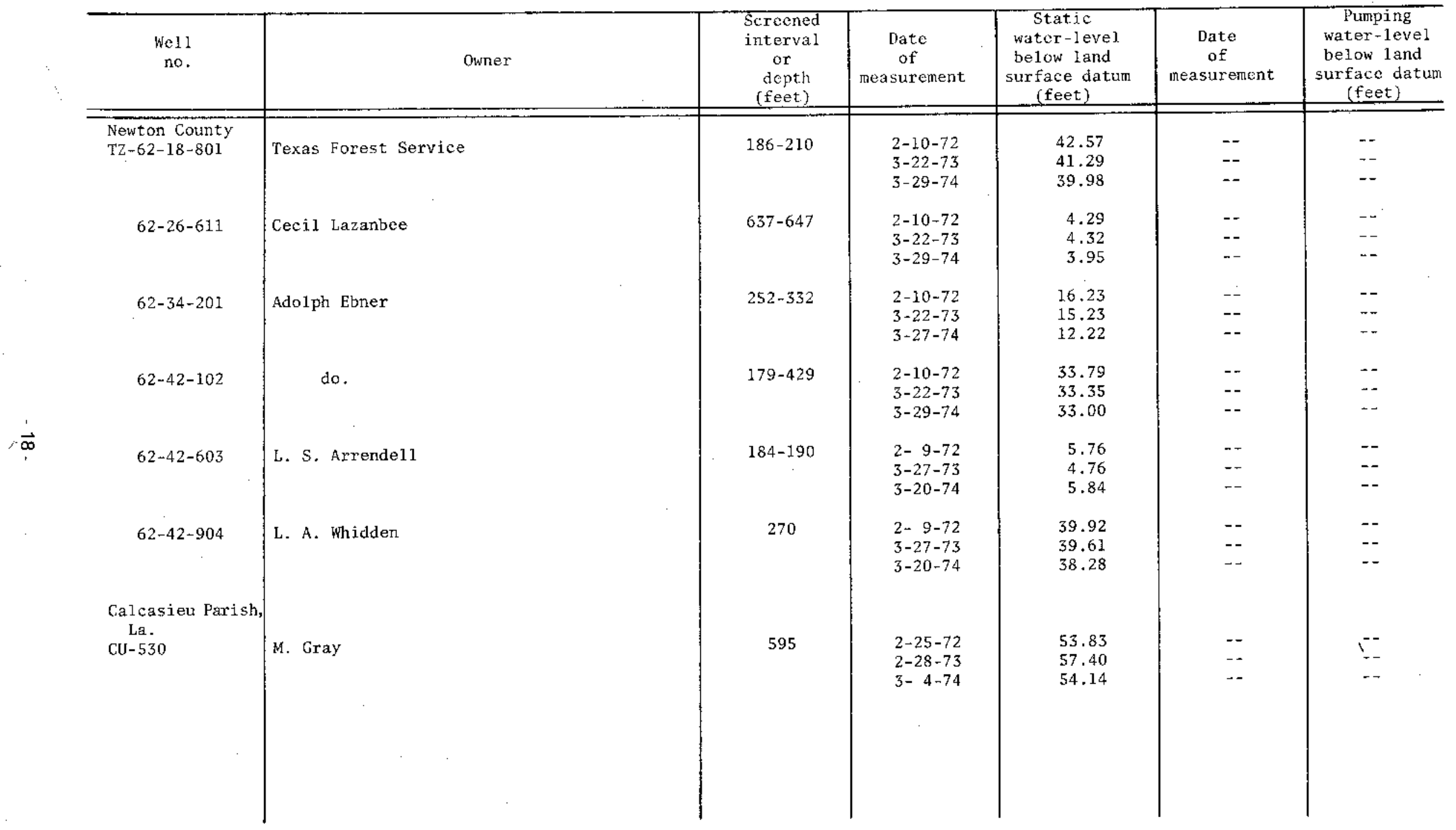


, 
Table 2.-Water-leve1 measurements In the Orange County area, 1971-74--Continued

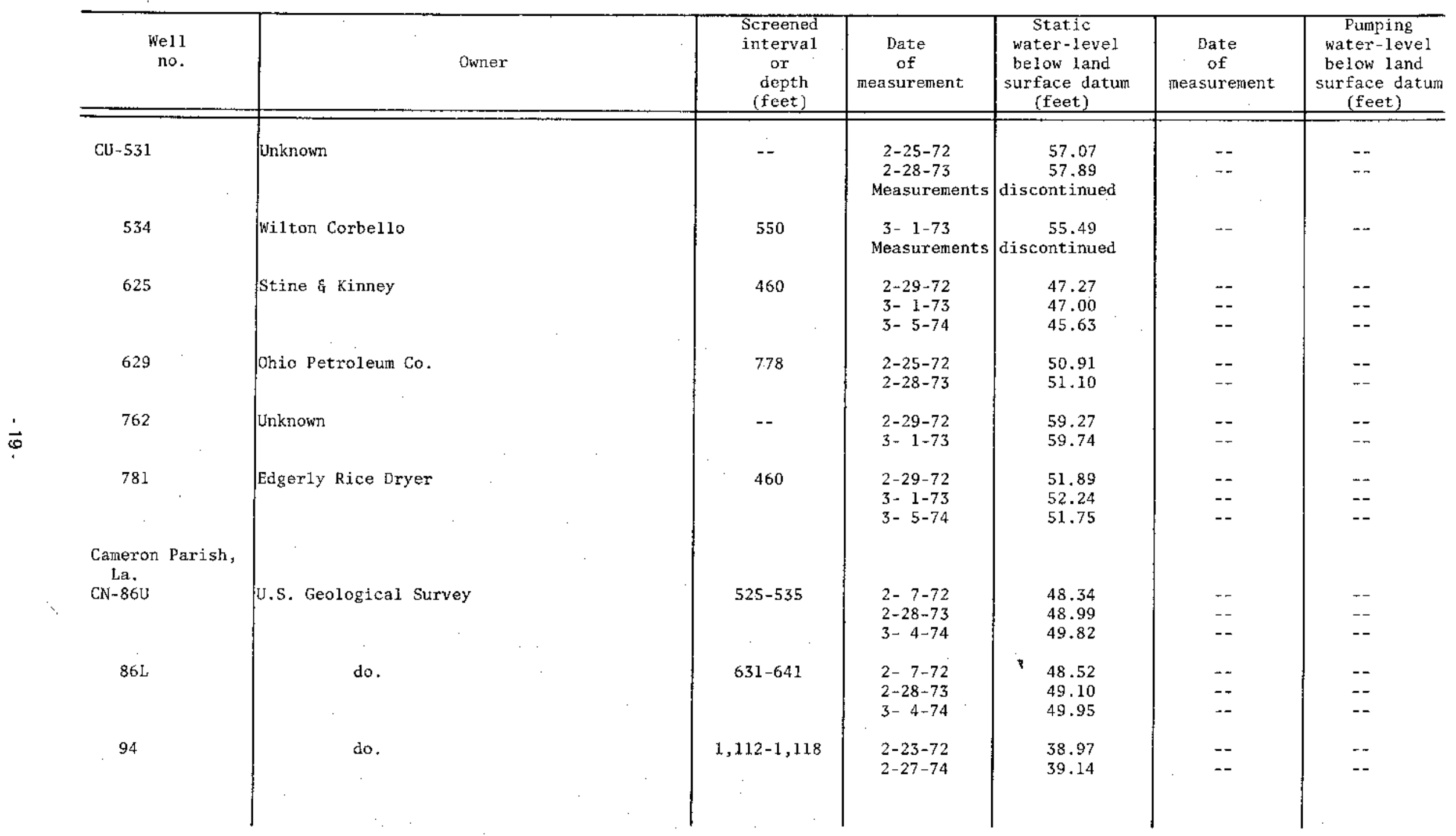


Table 3,--Chemical analyzee of water fron ure1ls 1o the dreoge County acea, 197l-74

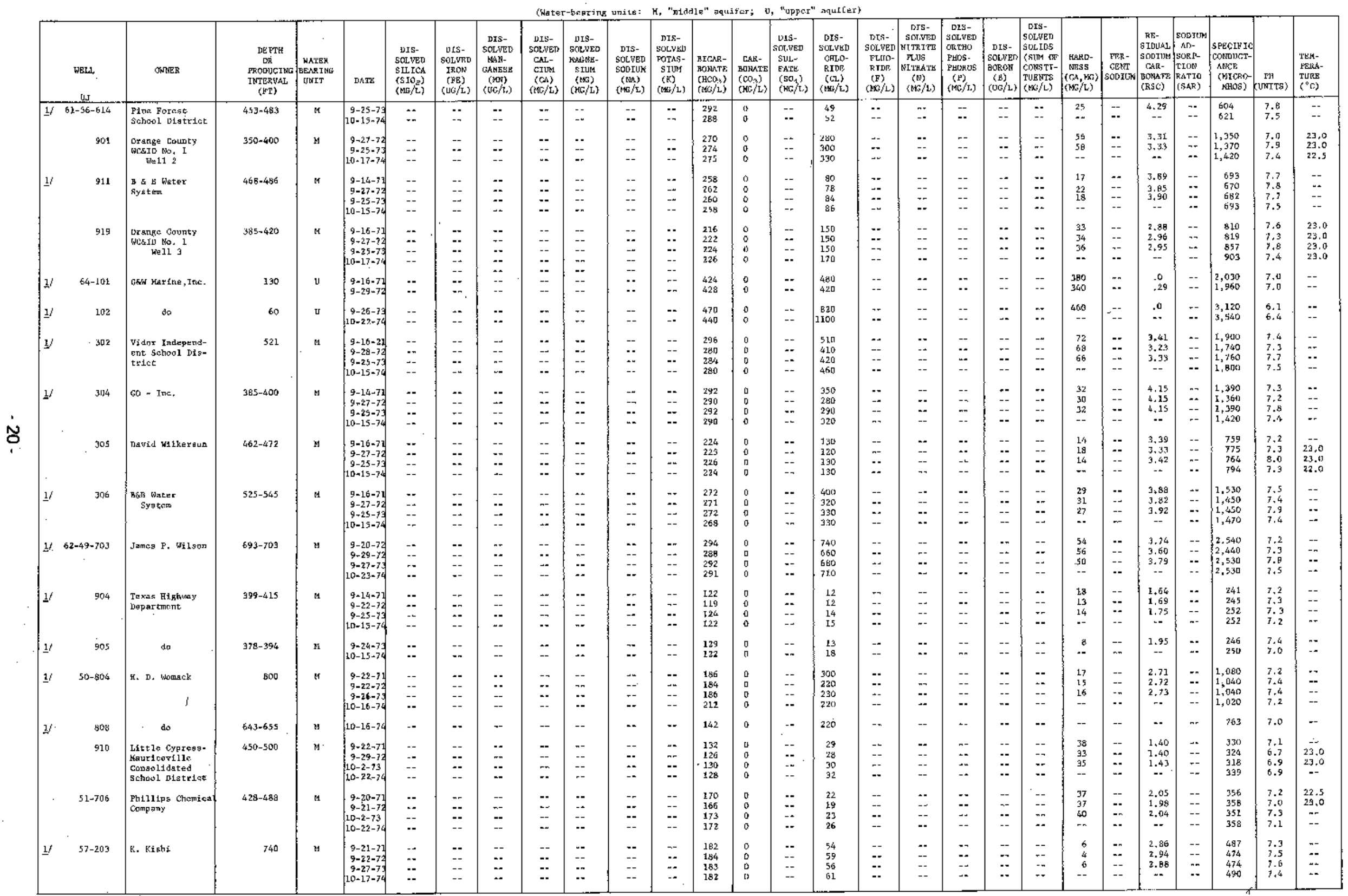





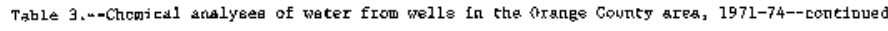

\begin{tabular}{|c|c|c|c|c|c|c|c|c|c|c|c|c|c|c|c|c|c|c|c|c|c|c|c|c|c|c|c|}
\hline $\begin{array}{c}\text { WRLL } \\
\text { US }\end{array}$ & OHEER & 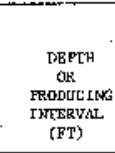 & 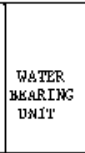 & DATE & 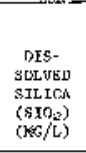 & 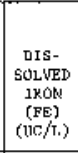 & 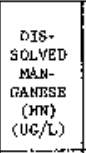 & 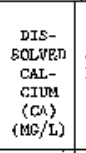 & 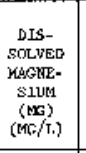 & 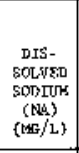 & 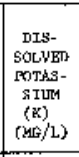 & 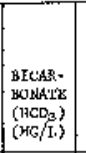 & 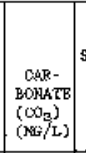 & 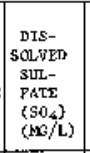 & 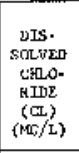 & 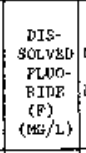 & 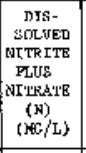 & 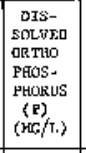 & 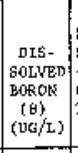 & 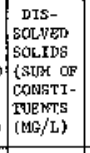 & 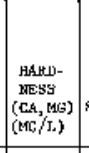 & 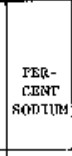 & 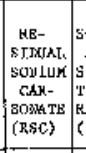 & 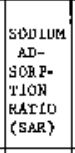 & 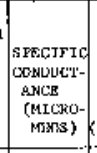 & 砶 & 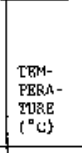 \\
\hline $62.57-401$ & 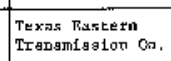 & $448-469$ & M & $\left|\begin{array}{l}9-14-71 \\
9-28-72 \\
9-28\end{array}\right|$ & $\because$ & 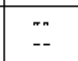 & $-\cdots$ & \begin{tabular}{|l|}
$-n$ \\
$-\pi$
\end{tabular} & \begin{tabular}{l|l}
- \\
\end{tabular} & $\because$ & $\because-$ & \begin{tabular}{|l|}
180 \\
178 \\
778
\end{tabular} & \begin{tabular}{|l|} 
\\
\\
0
\end{tabular} & $\because$ & $\begin{array}{l}63 \\
65 \\
64\end{array}$ & $\because$ & $\begin{array}{ll}- \\
\end{array}$ & $=-$ & $\because$ & $=$ & \begin{tabular}{|c|}
9 \\
10 \\
10
\end{tabular} & $\because-$ & $\begin{array}{l}2.77 \\
2.727 \\
2.72\end{array}$ & $\because$ & $\begin{array}{l}5105 \\
446\end{array}$ & 7.9. & 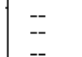 \\
\hline & & & & $\left|\begin{array}{c}9-27-73 \\
10-17-74\end{array}\right|$ & $\because$ & $\ddot{-}$ & $\because:$ & -- & -- & $\ddot{z}$ & $\because$ & $\begin{array}{l}\begin{array}{l}1871 \\
176\end{array} \\
276\end{array}$ & 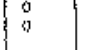 & $\because-$ & $\begin{array}{l}64 \\
68\end{array}$ & $\ddot{z}$ & $\because$ & $\overline{-}$ & -- & $\because$ & $\therefore$ & 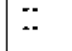 & $2, .77$ & $\because$ & $\begin{array}{l}444 \\
499\end{array}$ & $\begin{array}{l}7.8 \\
7.6\end{array}$ & $=$ \\
\hline 403 & 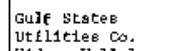 & 439-483 & к & $\left|\begin{array}{l}3-19-71 \\
9-27-77^{2}\end{array}\right|$ & -: & $\because$ & -- & $\because-$ & $\because$ & $\because$ & $\because$ & $\begin{array}{l}204 \\
199\end{array}$ & $\begin{array}{l}0 \\
0 \\
0\end{array}$ & $\because$ & $\begin{array}{l}240 \\
279\end{array}$ & $\because-$ & $\therefore$ & -- & 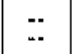 & -: & $4_{40}^{40}$ & - & $\begin{array}{l}2.54 \\
2.46\end{array}$ & $\because:$ & $\mid \begin{array}{l}1,080 \\
1,070\end{array}$ & 7.6 & 24.0 \\
\hline & $\mid$ & & & 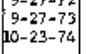 & $\because$ & $\because$ & $\because$ & $\because$ & -- & $\because:$ & $\because$ & $\begin{array}{l}2907 \\
210\end{array}$ & $\begin{array}{l}0 \\
0 \\
0\end{array}$ & 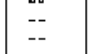 & $\begin{array}{l}280 \\
380 \\
390\end{array}$ & $\because$ & $\because-$ & -- & $\because-$ & $\because$ & ..- & - & $\begin{array}{l}2.37 \\
---\end{array}$ & -- & $\begin{array}{l}1,230 \\
1,440 \\
1,440\end{array}$ & 7.7 & $\begin{array}{l}24.0 \\
24.0\end{array}$ \\
\hline 404 & 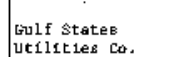 & $430-48 \mathrm{~K}$ & " & $\mid \begin{array}{c}9=-22-71 \\
9-27-77\end{array}$ & $\because$ & $\because-$ & -- & .- & -- & $\because$ & $\because$ & $\begin{array}{l}228 \\
236\end{array}$ & $:$ & $\ddot{z}$ & $\begin{array}{l}760 \\
840\end{array}$ & $\because-$ & -- & $\ddot{--}$ & $\because:$ & $\because$ & $\begin{array}{l}140 \\
180\end{array}$ & - & $\begin{array}{l}0.90 \\
0.31 \\
0.31\end{array}$ & $\because:$ & $\begin{array}{l}2,500 \\
2,690 \\
2,690\end{array}$ & $\begin{array}{l}7,2 \\
7,0\end{array}$ & \begin{tabular}{|l}
24.0 \\
24.0
\end{tabular} \\
\hline & vidor, kie11 i & & & $\left|\begin{array}{l}\mid 9-27-73 \\
10-2374\end{array}\right|$ & $\because$ & $\because$ & $\because$ & $-:$ & $=$ & $\ddot{-}$ & $\because$ & $\begin{array}{l}244 \\
246\end{array}$ & 0 & $\because-$ & $\begin{array}{r}950 \\
1,000\end{array}$ & $\ddot{z}$ & $\ddot{*}$ & $=$ & -- & $\because$ & 200 & $\because$ & 0.00 & $\because-$ & $\begin{array}{l}3,250 \\
3,320\end{array}$ & $\begin{array}{l}7.8 \\
7.2\end{array}$ & $\begin{array}{l}24.0 \\
24.5 \\
24.5\end{array}$ \\
\hline 405 & 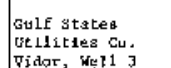 & $4,30-480$ & m & $10-23-74$ & -- & -- & .. & $-\cdot$ & .. & -- & -- & 220 & 0 & -. & 570 & -- & -. & .. & -- & -- & $-\cdot$ & -- & - & -- & 2,040 & 7.3 & 24.5 \\
\hline 406 & 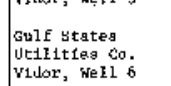 & $430-480$ & r & $10-23-74$ & -- & -- &.- & -. & -- & -- & - & 217 & 0 & -- & 560 & -- & -- & -- & -. & - & -. & -- & - & -- & 1,970 & 7.1 & 24.0 \\
\hline 407 & 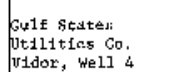 & $320-370$ & M & 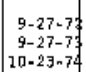 & $\because:$ & $\because:$ & $\because$ & $\because-$ & $\ddot{-}$ & 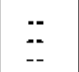 & $\because:$ & $\begin{array}{l}2100 \\
218 \\
211 \\
211\end{array}$ & $\begin{array}{l}0 \\
\vdots \\
0\end{array}$ & $\ddot{z}$ & $\begin{array}{l}65 \\
64 \\
68\end{array}$ & $\begin{array}{l}\because- \\
\because-\end{array}$ & $\ddot{-}$ & $\because:$ & $\begin{array}{l}\ddot{-} \\
\because-\end{array}$ & $\begin{array}{l}\cdots \\
--\end{array}$ & $\begin{array}{l}16 \\
13 \\
--\end{array}$ & $\because=$ & $\begin{array}{l}3.12 \\
3.31 \\
-. .-\end{array}$ & $\ddot{-}$ & $\begin{array}{l}5662 \\
565 \\
564\end{array}$ & $\begin{array}{l}7.4 \\
77.9 \\
7.4\end{array}$ & $\begin{array}{l}233.0 \\
23,0 \\
23.0\end{array}$ \\
\hline 408 & 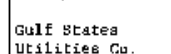 & 343-963 & " & $\left|\begin{array}{c}9-22-71 \\
4=27-77^{2}\end{array}\right|$ & - & $\therefore$ & .: & $\because:$ & - & .. & $\because$ & $=$ & $:$ & $\begin{array}{l}-. \\
--\end{array}$ & $\begin{array}{l}48 \\
45\end{array}$ & $\because$ & 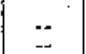 & $\because-$ & -- & -: & ${ }_{13}^{13}$ & $\because$ & $\begin{array}{l}3.84 \\
3.85\end{array}$ & $\because$ & $\begin{array}{l}5.95 \\
56 \%\end{array}$ & $\begin{array}{l}7.9 \\
7.6\end{array}$ & 23.0 \\
\hline & 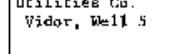 & & & 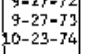 & $\ddot{-}$ & $\because$ & -- & -- & $=$ & $\because$ & $\because-$ & $\begin{array}{l}252 \\
250 \\
250\end{array}$ & $\begin{array}{l}0 \\
0 \\
0\end{array}$ & $\because$ & $\begin{array}{l}65 \\
77\end{array}$ & $\because-$ & -: & -- & $\ddot{--}$ & $=$ & 15 & - & 3.83 & $\because$ & $\begin{array}{l}619 \\
644\end{array}$ & $\begin{array}{l}8.1 \\
7.5\end{array}$ & 23.0 \\
\hline 501 & Flortida gas co. & $405-435$ & " & $\left\{\begin{array}{l}9-21-71 \\
9-27-72,\end{array}\right\}$ & $\ddot{-}$ & 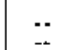 & $\because-$ & $\because$ &.- & $\because$ & $\because$ & $\begin{array}{l}173^{\circ} \\
171\end{array}$ & 0 & $\because$ & $\begin{array}{l}28 . \\
26\end{array}$ & $\because$ & $\because$ & $\because-$ & -- & $\ddot{--}$ & 11 & -- & $\begin{array}{l}2.70 \\
2.58 \\
2.57\end{array}$ & $\because$ & $\begin{array}{l}376 \\
371\end{array}$ & 7.5 & $\ddot{-}$ \\
\hline & & & & $\mid \begin{array}{l}3-27-73 \mid \\
10-17-74\end{array}$ & -- & $=$ & $\because$ & $\ddot{-}$ & $=$ & $\because-$ & $\because$ & $\begin{array}{l}175 \\
179\end{array}$ & 10 & $\ldots$ & $\begin{array}{l}28 \\
30\end{array}$ & $\overline{-}$ & $\because:$ & $\ddot{-}$ & $\because-$ & $\because-$ & 8 & $\because$ & $\begin{array}{l}2.71 \\
.-\end{array}$ & $\because$ & $\begin{array}{l}376 \\
375\end{array}$ & 7.74 & $\because-$ \\
\hline 302 & The Texse co. & $478-52 \mathrm{~B}$ & к & $\left|\begin{array}{l}g=21-71 \\
9=22-72\end{array}\right|$ & $\because$ & $\therefore$ & -. & $\because$ & - & $\because$ & $=$ & \begin{tabular}{|l}
188 \\
199
\end{tabular} & $\begin{array}{l}0 \\
n \\
n\end{array}$ & $=$ & $\begin{array}{l}30 \\
28\end{array}$ & $\because$ & $\because$ & $\because$ & $\because$ & -- & $\begin{array}{l}10 \\
11 \\
a\end{array}$ & $\because$ & 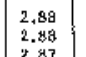 & $\because-$ & $\begin{array}{l}404 \\
397\end{array}$ & $\begin{array}{l}7.5 \\
7.6 \\
7.6\end{array}$ & $\because:$ \\
\hline & & & & $\left|\begin{array}{l}9-27-73 \\
20-17-74\end{array}\right|$ & $\ddot{-}$ & $\ddot{x}$ & $\ddot{-}$ & $\because$ & -- & $\because$ & $\because$ & 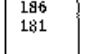 & 10 & $\because$ & 24 & $\ddot{--}$ & $\because$ & $\because$ & $\ddot{--}$ & $\because$ & $\therefore$ & $\ddot{--}$ & & $\ddot{*}$ & 371 & 7,5 & -- \\
\hline 605 & Fand Cormier & $469-489$ & " & 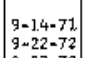 & $\because-$ & $\therefore$ & 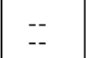 & $\because:$ & $\because$ & - & $\because-$ & $\begin{array}{l}144 \\
1444\end{array}$ & $:$ & -- & $\begin{array}{l}24 \\
22 \\
22\end{array}$ & $\because$ & -- & -- & $\because$ & $=$ & ${ }_{8}^{11}$ & $\because-$ & $\begin{array}{l}2.14 \\
2.20 \\
2.20\end{array}$ & $\because$ & $\begin{array}{l}321 \\
325 \\
322\end{array}$ & $\begin{array}{l}7.4 \\
7.2\end{array}$ & $\because$ \\
\hline & & & & $\left|\begin{array}{l}9.2-27-77 \\
10-17-74\end{array}\right|$ & -- & $\because$ & $\because$ & $\because$ & $\ddot{-}$ & $\because$ & $\because$ & 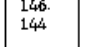 & $:$ & $\ddot{z}$ & $\begin{array}{l}26 \\
26\end{array}$ & $\ddot{--}$ & $\because$ & $\because$ & -- & $\ddot{--}$ & $\therefore$ & $=$ & 2.23 & $\ddot{--}$ & \begin{tabular}{|l|}
322 \\
332
\end{tabular} & $\begin{array}{l}7.4 \\
7.2\end{array}$ & -- \\
\hline $900 t$ & 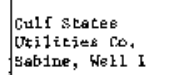 & $575-625$ & M & $\mid 9-28-72$ & -- & -. & - & -- & -- & -. & -. & 222 & 0 & -. & 910 & -- & -- & -. & $\cdots$ & -- & $140^{\circ}$ & $\cdots$ & .76 & 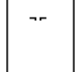 & 3,110 & 6.8 &.- \\
\hline 904 & 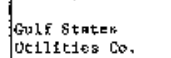 & $432-455$ & " & $\left|\begin{array}{l}9-21-71 \\
9-28-72\end{array}\right|$ & 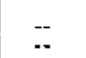 & 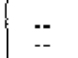 & 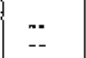 & $\because$ & -- & 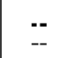 & $\because$ & $\begin{array}{l}2200 \\
220\end{array}$ & $\begin{array}{l}0 \\
0 \\
0\end{array}$ & $\because$ & $\begin{array}{l}40 \\
39\end{array}$ & - & -- & $\ddot{--}$ & $\therefore$ & $\because$ & $\begin{array}{l}10 \\
11 \\
11\end{array}$ & -- & $\begin{array}{l}3.41 \\
3.39 \\
3.34\end{array}$ & $\because$ & $\begin{array}{l}4912 \\
492\end{array}$ & $\begin{array}{l}7.6 \\
7.5\end{array}$ & $\begin{array}{l}24.0 \\
24.0 \\
240\end{array}$ \\
\hline & Bablna, Кc11 4 & & & 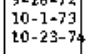 & $\because$ & $\because$ & $=$ & -- & $\because$ & $\because:$ & $\because$ & $\begin{array}{l}222 \\
224\end{array}$ & $\begin{array}{l}0 \\
0 \\
0\end{array}$ & $\because$ & $\begin{array}{l}42 \\
45\end{array}$ & & $\because:$ & $\because$ & $\overline{-}$ & $\because$ & $\stackrel{11}{--}$ & $\because:$ & 3,42 & $\because-$ & $\begin{array}{l}{ }^{199} \\
500\end{array}$ & 7.7 & $\begin{array}{l}24.0 \\
24.0\end{array}$ \\
\hline 905 & $\begin{array}{l}\text { culf States } \\
\text { vut11t1tes co. }\end{array}$ & $422-461$ & " & $\left|\begin{array}{l}9-21-71 \\
9-28-72\end{array}\right|$ & $\because$ & $\because$ & $\because-$ & $\because$. & $\ddot{z}$ & $=$ & $\therefore$ & $\begin{array}{l}245 \\
249\end{array}$ & $\begin{array}{l}n \\
0 \\
0\end{array}$ & $\because$ & $\begin{array}{l}43 \\
40\end{array}$ & $\ddot{-}$ & $\because-$ & -- & $\because$ & $\because$ & $\begin{array}{l}13 \\
1,3\end{array}$ & -- & \begin{tabular}{|l|l|}
9.76 \\
3.82
\end{tabular} & $\because$ & $\begin{array}{l}535 \\
527 \\
521\end{array}$ & $\begin{array}{l}7.7 \\
7.6\end{array}$ & $\because$ \\
\hline & Sabtites, weII's & & & $\left|\begin{array}{l}10-1-1,3] \\
10-23-74\end{array}\right|$ & $z$ & $\because$ & $\therefore$ & $=$ & $=$ & $\because$ & $\because$ & $\begin{array}{l}250 \\
250\end{array}$ & $\begin{array}{l}0 \\
0 \\
0\end{array}$ & 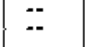 & $\begin{array}{l}44 \\
46\end{array}$ & $=$ & 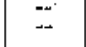 & $\because$ & $\because$ & -- & ${ }^{13} \cdot$ & $\ddot{-}$ & $=3 .-64$ & $\because-$ & $\begin{array}{l}541 \\
543\end{array}$ & 7.8 & $\begin{array}{l}23.5 \\
23.5\end{array}$ \\
\hline 907 & 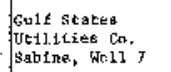 & 604-654 & $\mathrm{M}$ & $0-24-74$ & - & .- &.- & .- & -- & -- & - & 191 & 0 & -- & w10 & -. & -- & -. & -. & -- &.- & -. & $\cdots$ & -- & 646 & 7.5 & 25.0 \\
\hline 908 & 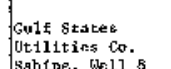 & $573-623$ & M & $10-24-$ & -- & .. & -. & - & $\therefore$ & -. & .. & 240 & ${ }^{\circ}$ & .. & 46 & 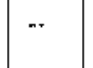 & -- & -- & $\cdot-$ & -- & -- & -- & -- & -. & 527 & 7.5 & 24.0 \\
\hline $56-304$ & 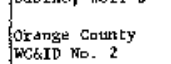 & $626-706$ & "M & $\mid \begin{array}{c}9-16-717 \\
9=-20772\end{array}$ & $\because$ & $\because$ & $\because-$ & $\because$ & $\therefore$ & -- & - & $\begin{array}{l}\begin{array}{l}190 \\
188\end{array} \\
189\end{array}$ & $:$ & $\because$ & $\begin{array}{l}200 \\
190\end{array}$ & $\because$ & $\because$ & $\ddot{-}$ & -- & $\because-$ & $\begin{array}{l}62 \\
64\end{array}$ & $\because-$ & $\begin{array}{l}1.87 \\
1.80\end{array}$ & -- & $\begin{array}{l}928 \\
910\end{array}$ & 7.12 & $\because$ \\
\hline & & & & $\begin{array}{l}90-26-73 \\
10-16-74\end{array}$ & $\because$ & $\because$ & $\because$ & $\ddot{-}$ & $\ddot{--}$ & $\cdots$ & $\because-$ & $\begin{array}{l}193 \\
192\end{array}$ & $\begin{array}{l}\pi \\
0\end{array}$ & 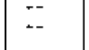 & $\begin{array}{l}\begin{array}{l}190 \\
180\end{array} \\
180\end{array}$ & & & $\because$ & $\because$ & $\because$ & $\stackrel{-2}{--}$ & 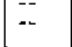 & $\because, 92$ & $\because$ & 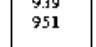 & $\begin{array}{l}7.4 \\
7.2\end{array}$ & $\ddot{z}$ \\
\hline 305 & Cyty of orange & $520-610$ & M & $\begin{array}{l}9-19-72 \\
9,-24-73 \\
10-16\end{array}$ & $\because$ & $\begin{array}{l}-- \\
\because\end{array}$ & $=-$ & $\because$ & $\ddot{z}$ & $\because$ & -- & $\begin{array}{l}160 \\
165 \\
164\end{array}$ & $\begin{array}{l}0 \\
0 \\
0 \\
0\end{array}$ & $\because$ & $\begin{array}{l}99 \\
710 \\
1120 \\
120\end{array}$ & $\because-$ & $\ddot{-}$ & $\begin{array}{l}\therefore- \\
\therefore-\end{array}$ & $=$ & $\ddot{-}$ & $\begin{array}{l}75 \\
8 \%\end{array}$ & $=$ & $\begin{array}{l}1,12 \\
1,0,06 \\
-.:\end{array}$ & $\because-$ & $\begin{array}{l}609 \\
649 \\
660\end{array}$ & $\left\{\begin{array}{l}6.6 \\
7.6 \\
7.0\end{array}\right.$ & $\begin{array}{l}\begin{array}{l}24.0 \\
24.0 \\
24.0\end{array} \\
24.0\end{array}$ \\
\hline
\end{tabular}



Tab1e 3.-Chaterical analyges of water froul wells in tha Otange county area, 1971-7it--cont1nued

\begin{tabular}{|c|c|c|c|c|c|c|c|c|c|c|c|c|c|c|c|c|c|c|c|c|c|c|c|c|c|c|c|}
\hline orut & OMERR & 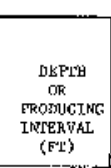 & 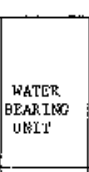 & DATs & 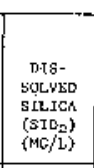 & 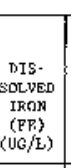 & 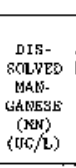 & 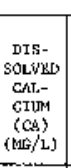 & 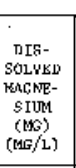 & 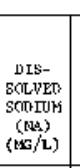 & 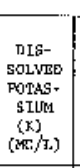 & 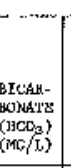 & 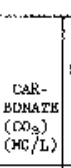 & 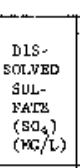 & 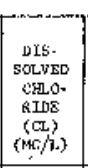 & 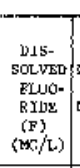 & 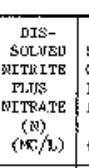 & 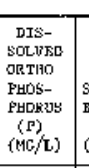 & & & 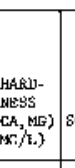 & 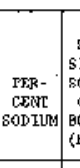 & 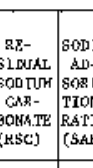 & 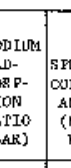 & 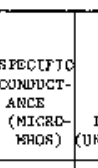 & 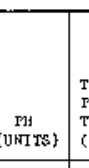 & 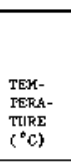 \\
\hline 62-58-324 & Sity of Pinnthurst & $365-44.5$ & " & & $=$ & $\because:$ & $\because$ & $\because$ & $=$ & $\because$ & $\because$ & $\begin{array}{l}1489 \\
143\end{array}$ & $\stackrel{\circ}{0}$ & $\because=$ & 䛼1 & $\because-$ & $=$ & $\because$ &.- & $\because$ & $\frac{38}{36}$ & $\because$ & & & $\begin{array}{l}294 \\
295 \\
295\end{array}$ & & $\begin{array}{l}23.0 \\
23.0\end{array}$ \\
\hline & & & & & $\because$ & $\because$ & $\because-$ & -- & 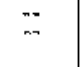 & $\because:$ & $\ddot{*}$ & 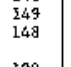 & $:$ & $\because-$ & 醋 & $\because$ & $\because:$ & $\ddot{*}$ & $\because$ & $\because:$ & 保. & $\because-$ & & & 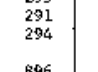 & 7. & $\begin{array}{l}23.0 \\
23.0 \\
20.5\end{array}$ \\
\hline 325 & 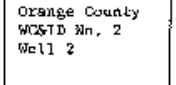 & 620.650 & x & 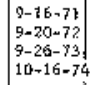 & $\because \because$ & $\ddot{z}$ & $\ddot{z}$ & $\because:$ & $\because:$ & $\ddot{\because}$ & $\ddot{\because}$ & $\begin{array}{l}1960 \\
1969 \\
1992 \\
190\end{array}$ & $\begin{array}{l}0 \\
0 \\
0 \\
0\end{array}$ & $\because:$ & 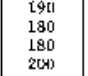 & $\ddot{\ddot{z}}$ & $\ddot{z}$ & $\ddot{\because}$ & $\because:$ & $\ddot{\because}$ & $\begin{array}{l}51 \\
52 \\
5.4\end{array}$ & :-: & $\begin{array}{l}2.09 \\
2.01 \\
2.07 \\
2.07\end{array}$ & $\Xi$ & 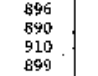 & $\begin{array}{l}7.2 \\
7.0 \\
7.6 \\
7.2\end{array}$ & $\begin{array}{l}245.5 \\
25: 0 \\
25.9 \\
24.5\end{array}$ \\
\hline 402 & 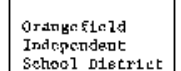 & 515-535 & " & 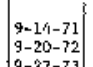 & $\because:$ & $\because$ & :- & $\because$ & $\because$ & $\therefore$ & $\because:$ & 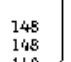 & $:$ & $\therefore$ & $\begin{array}{c}26 \\
26 \\
26\end{array}$ & $=$ & $\because:$ & $\because:$ & $\because$ & $\because:$ & $\begin{array}{l}6 \\
6 \\
6\end{array}$ & :. & $\begin{array}{l}2.31 \\
2,31\end{array}$ & $\because$ & $\begin{array}{l}341 \\
3,39\end{array}$ & $\begin{array}{l}7.6 \\
7.3\end{array}$ & $\because=$ \\
\hline 4009 & J. א. Fht Fl1 pes & 5664.651 & & & -- & - & $\because=$ & $\because$ & $\because$ & $\because$ & $\because$ & 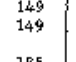 & $:$ & $\because$ & $\begin{array}{l}25 \\
3_{25}\end{array}$ & $\because$ & $\because-$ & $\because=$ & $=$ & $\because$ & $\therefore$ & $=$ & . & & 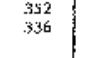 & 7.5 .5 & $\because$ \\
\hline & raterer speten & & $n$ & & $\because=$ & $\ddot{z}$ & $\because$ & $\because=$ & $\ddot{z}$ & $\because:$ & $\ddot{z}$ & 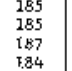 & $\begin{array}{l}0 \\
\vdots \\
0 \\
0\end{array}$ & $\because$ & 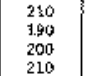 & $\because:$ & $\because:$ & $\because \because$ & $\because:$ & $\ddot{z}$ & $\begin{array}{l}22 \\
24 \\
25 \\
25\end{array}$ & $\because:$ & $\begin{array}{l}2.59 \\
2.55 \\
2,56 \\
2,56\end{array}$ & $\because$ & 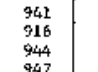 & $\begin{array}{l}7.4 \\
7.2 .3 \\
7.3\end{array}$ & $\because$ \\
\hline $4 x$ & 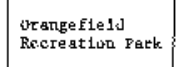 & $460-450^{\circ}$ & м & $\begin{array}{l}9=21-71 \\
9-20-72\end{array}$ & $\because:$ & $\ddot{-}$ & $\because-$ & $\because$ & $\because$ & 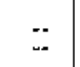 & 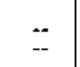 & $\begin{array}{l}232 \\
230\end{array}$ & $: 0$ & -- & ${ }_{14}^{18}$ & $\because:$ & $\because$ & $\ddot{-}$ & $\because$ & $\because-$ & ${ }_{10}^{11}$ & -: & $\begin{array}{l}3.56 \\
3.57\end{array}$ & $\because-$ & $\begin{array}{l}430 \\
433 \\
435\end{array}$ & 7,6 & $\because$ \\
\hline & 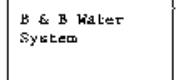 & $208-218$ & 0 & 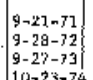 & $\because$ & $\because:$ & $\because:$ & $\because \because$ & $\because$ & $\because:$ & $\because:$ & 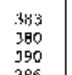 & $\begin{array}{l}0 \\
: \\
0\end{array}$ & $\because \because$ & $\begin{array}{l}\frac{700}{55} \\
64 \\
64\end{array}$ & $\ddot{\ddot{z}}$ & $\because:$ & $\because$ & $\because:$ & $\because:$ & 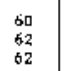 & $\ddot{z}$ & $\begin{array}{l}5.08 \\
\text { s.9. } \\
5.15 \\
\end{array}$ & $\because$ & $\begin{array}{l}799 \\
796 \\
809\end{array}$ & $\begin{array}{l}7.4 \\
7.2 \\
r .2\end{array}$ & $\because$ \\
\hline 513 & 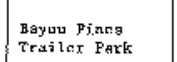 & $205-215$ & v & $10-23-74$ & -- & -- & - & - & .. & - & -- & 378 & " & $\ldots$ & 6.5 & -- & - & -- & -- & $\cdots$ & -. & .. &.- &. & $\begin{array}{l}802 \\
883\end{array}$ & $\begin{array}{l}7.4 \\
7.4\end{array}$ & $\ddot{-}$ \\
\hline & Doonarar Corpe. & $\infty 00$ & n & 6.711 & -. & -- & .. & .. & -. & - & - & 180 & 0 & .. & 150 & -- & -- & .. &. & -. & 47 & .. & 2.01 & .. & 727 & 7.1 & .. \\
\hline & 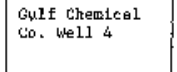 & $604-717$ & " & 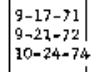 & $\because \because$ & $\ddot{-}$ & $\because:$ & $\because:$ & $\because:$ & $\because:$ & $\because:$ & $\begin{array}{l}200 \\
196 \\
218\end{array}$ & $\begin{array}{l}0 \\
\vdots \\
0\end{array}$ & $\because:$ & 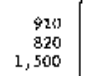 & $\because:$ & $\because:$ & $\because$ & $\because$ & $\because-$ & $\begin{array}{l}170 \\
170 \\
180 \\
-\pi\end{array}$ & $\because$ & \begin{tabular}{l|}
$: 0$ \\
$:-0$ \\
$:-1$
\end{tabular} & $=$ & 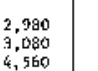 & $\begin{array}{l}6.9 .9 \\
6.7 \\
r .4\end{array}$ & 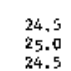 \\
\hline 606 & $\begin{array}{l}\text { collf chenticas } \\
\text { co. wel1 } \mathrm{s}\end{array}$ & $630-712$ & n & 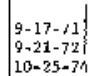 & $\because$ & 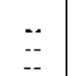 & $\because$ & $\ddot{z}$ & $\because:$ & $\because$ & $\because:$ & 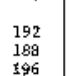 & $:$ & $\ddot{z}$ & $\begin{array}{l}770 \\
7>0 \\
200 \\
200\end{array}$ & 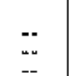 & $\ddot{z}$ & $\because:$ & $\because$ & $\because$ & $\begin{array}{l}30 \\
32 \\
32\end{array}$ & 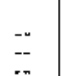 & 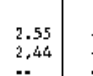 & $=$ & $\begin{array}{l}827 \\
871 \\
871\end{array}$ & $\begin{array}{l}7.4 \\
6.9\end{array}$ & $\begin{array}{l}25,0 \\
25,0 \\
25,0\end{array}$ \\
\hline 608 & 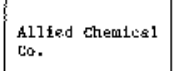 & 620-735 & " & 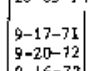 & $\because:$ & :- & $\because$. & $=$ & $=$ & $\because$ & $\because:$ & 20 & $:$ & - & 年 1200 & $\because=$ & $\because$ & 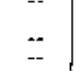 &.$:$ & $\ddot{z}$ & 37 & 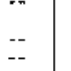 & $\begin{array}{l}2.67 \\
2.40 \\
20.40\end{array}$ & $\because$ & 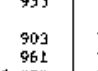 & 7.1 .5 & $\begin{array}{l}25.3 \\
25.0 \\
25.0\end{array}$ \\
\hline & & & & & $\because-$ & $\because-$ & $\because-$ & $\because:$ & $\because:$ & $\because$ & $\because=$ & $\begin{array}{l}212 \\
\text { tid }\end{array}$ & 0 & $\because$ & $\begin{array}{l}220 \\
2200 \\
220\end{array}$ & $\because-$ & $\because-$ & $\because$ & $\because$ & $\because$ & 52 & $\because$ & 2.43 & $\because:$ & , 1, 1,200 & 7.2 .7 & \\
\hline 609 & 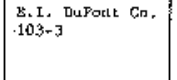 & 6344-723 & ${ }^{\mathrm{s}}$ & 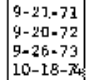 & $\because \because$ & $\because$ & $\because:$ & $\because:$ & $\because$ & $\ddot{z}$ & $\because:$ & $\begin{array}{l}\frac{198}{2190} \\
198 \\
200\end{array}$ & $\begin{array}{l}: \\
\vdots \\
0\end{array}$ & 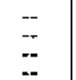 & $\begin{array}{l}190 \\
190 \\
2120 \\
220\end{array}$ & $\ddot{z}$ & $\because:$ & $\ddot{z}$ & 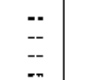 & $\ddot{z}$ & $\begin{array}{l}\text { s. } \\
52 \\
52 \\
52\end{array}$ & $\because:$ & $\begin{array}{l}2.23 \\
2.24 \\
2.24 \\
2.24\end{array}$ & $\because$ & 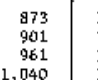 & $\begin{array}{l}7.3 \\
7,4.4 \\
i, 3\end{array}$ & $\begin{array}{l}25.0 \\
25: 0 \\
25: 0 \\
25: 0\end{array}$ \\
\hline 612 & 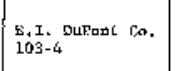 & 735 & n & 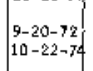 & $\because:$ & $\because:$ & $\because:$ & $\because$ & $\because-$ & $\because:$ & $\because$ & $\begin{array}{l}198 \\
206\end{array}$ & $:$ & $\because$ & ${ }_{270}^{160}$ & :: & $\because$. & $\because$ & $\because$ & $\because$ & 58 & $\because$ & 2.09 & $=$ & 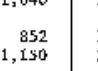 & $\begin{array}{ll}7.1 \\
7.2\end{array}$ & $\begin{array}{l}25.0 \\
25\end{array}$ \\
\hline 6.4 & 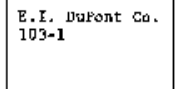 & 726 & $"$ & & & $\because:$ & $\because:$ & $\because:$ & $\ddot{\because}$ & $\because=$ & $\because=$ & 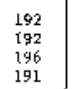 & $\begin{array}{l}0 \\
: \\
0 \\
0\end{array}$ & $\begin{array}{l}\ddot{-} \\
--\end{array}$ & $\begin{array}{l}170 \\
180 \\
200 \\
240\end{array}$ & $\begin{array}{l}\ddot{-} \\
\because\end{array}$ & $\ddot{\because}$ & $\ddot{:}$ & $\because:$ & $\because:$ & $\begin{array}{l}43 \\
65 \\
63 \\
--\end{array}$ & $\begin{array}{l}\ddot{*} \\
\cdots \\
\cdots\end{array}$ & $\begin{array}{l}2.29 \\
1.85 \\
1.95 \\
-2.95\end{array}$ & & 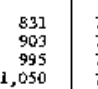 & $\begin{array}{l}7.2 \\
7,2.2 \\
7, .5 \\
i, 3\end{array}$ & $\begin{array}{l}25.0 \\
25.0 \\
25.0 \\
25.0\end{array}$ \\
\hline 615 & 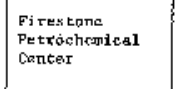 & $611-700$ & " & & & $\begin{array}{l}-= \\
-\end{array}$ & 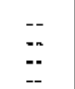 & $\because:$ & $\ddot{z}$ & $\begin{array}{l}\because: \\
\because:-\end{array}$ & $\begin{array}{l}\because \\
\because\end{array}$ & 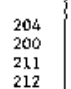 & $\begin{array}{l}0 \\
: \\
0 \\
0 \\
0\end{array}$ & $\ddot{z}$ & $\begin{array}{l}770 \\
730 \\
620 \\
8 \neq 0 \\
\text { sto }\end{array}$ & $\begin{array}{l}-- \\
\because:\end{array}$ & 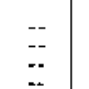 & $=$ & $\because:$ & $\because:$ & $\begin{array}{l}1100 \\
\text { s. } \\
:--\end{array}$ & 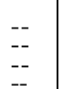 & 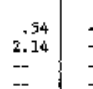 & & $\begin{array}{l}2,540 \\
2,540 \\
2,700 \\
2,400\end{array}$ & $\begin{array}{l}7.1 \\
6.97 \\
i .3\end{array}$ & $\begin{array}{l}24.5 \\
25.5 \\
24.0 \\
24.5\end{array}$ \\
\hline 616 & 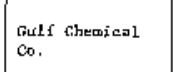 & 718 & " & $10-25-74$ & .- & .. & - & - & - &. & - & 184 & 0 & $\cdots$ & $\begin{array}{l}0.000 \\
1,000\end{array}$ & - & - & -- & -- &. & $\cdots$ & - & &.- & 3,170 & 7.4 & $\begin{array}{l}24.5 \\
24.0\end{array}$ \\
\hline & A. schut tuan con. & $440-460$ & $s$ & & $\ddot{:-}$ & $\ddot{\because}$ & $\because$ & $\because$ & $\ddot{z}$ & $\because:$ & $\because$ & 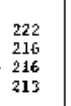 & $:$ & $\because$ & $\begin{array}{l}16 \\
14 \\
18 \\
19\end{array}$ & $\because$ & $\because$ & $\begin{array}{l}\because- \\
\because\end{array}$ & $\because$ & $\because$ & $\begin{array}{l}18 \\
24 \\
20 \\
--\end{array}$ & $\ddot{\because}$ & 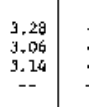 & 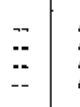 & $\begin{array}{l}413 \\
\begin{array}{l}413 \\
404 \\
404 \\
400\end{array}\end{array}$ & $\begin{array}{l}7.8 \\
7.8 \\
7.5 \\
7.5\end{array}$ & $\ddot{z}$ \\
\hline 629 & 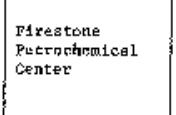 & ${ }^{595-680}$ & " & 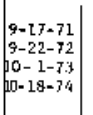 & $\because=$ & $\because$ & $\ddot{z}$ & $\because$ & $\ddot{z}$ & $\begin{array}{l}\ddot{-} \\
\because:\end{array}$ & $\because:$ & $\begin{array}{l}128 \\
187 \\
192 \\
192 \\
192\end{array}$ & 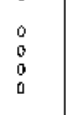 & $\because$ & $\begin{array}{l}190 \\
190 \\
220 \\
270 \\
270\end{array}$ & $\because=$ & $\because \because$ & $\equiv$ & $\because$ & & $\begin{array}{l}32 \\
32 \\
42 \\
-12\end{array}$ & $\because:$ & $\begin{array}{l}2.44 \\
2.42 \\
2.31 \\
-2.31\end{array}$ & \begin{tabular}{l|l}
$:$ & $\mid$ \\
$:$ & 1, \\
1,
\end{tabular} & 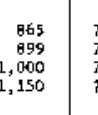 & $\begin{array}{l}7.2 \\
7.2 \\
7.5 \\
7,1\end{array}$ & $\begin{array}{l}\begin{array}{l}24.5 \\
25.5 \\
24.5 \\
24.5\end{array} \\
24.5\end{array}$ \\
\hline
\end{tabular}

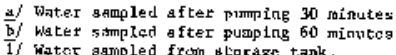




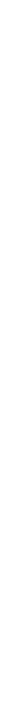


Table 3.--Lhemical analyses of vater from wella 1n the orange County area, 1971-74--continued

\begin{tabular}{|c|c|c|c|c|c|c|c|c|c|c|c|c|c|c|c|c|c|c|c|c|c|c|c|c|c|c|c|}
\hline vatit & ander & 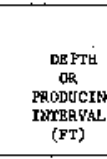 & 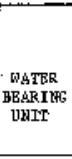 & DARE & 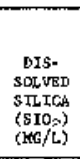 & 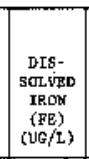 & 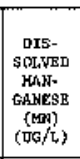 & 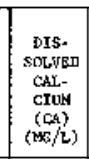 & 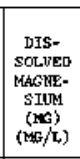 & 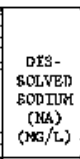 & 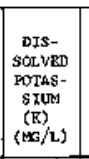 & 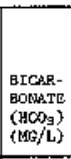 & 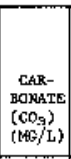 & 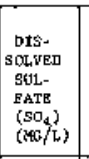 & 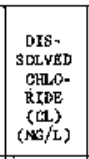 & 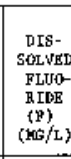 & 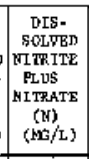 & 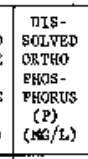 & 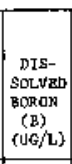 & 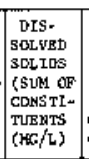 & 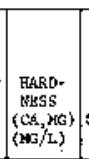 & 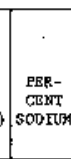 & & 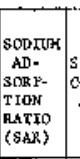 & 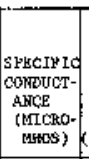 & pourtss & 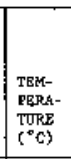 \\
\hline 62-53-631 & 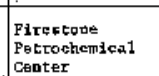 & $\begin{array}{l}5955-625 \\
6355-650 \\
6\end{array}$ & " & 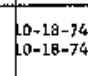 & $\frac{y}{y^{\prime}}=$ & $\because$ & $\because-$ & $\because$ & $\because$ & $=$ & $\because$ & \begin{tabular}{|l}
220 \\
222 \\
222
\end{tabular} & $:$ & $\because$ & $\mid \begin{array}{c}830 \\
850 \\
850\end{array}$ & $\because$ & $\because$ & $\because$ & $=$ & $\because$ & $\because$ & $=$ & $\because$ & $\because=z_{2}^{2}$ & $\mid$ & $\begin{array}{l}7.3 \\
.2 .2\end{array}$ & $\begin{array}{l}24.0 .0 \\
24.5\end{array}$ \\
\hline 638 & 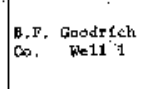 & 640,7730 & " & 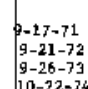 & $\because$ & $\because:$ & $\because=$ & $\because=$ & $\ddot{z}$ & $\because$ & $\because:$ & 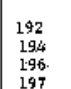 & $\begin{array}{l}0 \\
0 \\
0 \\
0\end{array}$ & $\because=$ & 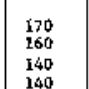 & $\because$ & $\ddot{\because}$ & $\because$ & $\ddot{\because}$ & $\because$ & $\begin{array}{l}31 \\
40 \\
42 \\
-1\end{array}$ & $\therefore$ & 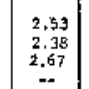 & $\because \because$ & 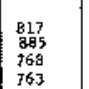 & $\begin{array}{l}7.4 \\
7.2 \\
3.2 \\
8.4\end{array}$ & $\begin{array}{l}25.0 \\
\text { 25:0 } \\
25.5 \\
24.5\end{array}$ \\
\hline 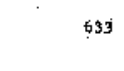 & 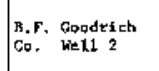 & $625-725$ & r. & $\mid \begin{array}{l}9-20-71 \\
9-21-77 \\
-21-7\end{array}$ & $\because$ & $\because$ & $\because$ & $\because$ & $\because$ & $\therefore$ & $\because=$ & $\begin{array}{l}208 \\
200 \\
2112 \\
212\end{array}$ & $\begin{array}{l}0 \\
0 \\
0 \\
0\end{array}$ & $\because$ & $\begin{array}{l}340 \\
\text { 250 } \\
\text { 1200 }\end{array}$ & $\because$ & $=$ & $\because$ & $\because$ & $\therefore$ & $\begin{array}{l}35 \\
5.5 \\
-.\end{array}$ & $=$ & $\left|\begin{array}{l}2.72 \\
2.32 \\
-.=6\end{array}\right|$ & $\because$ & 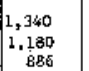 & $\mid \begin{array}{l}7.12 \\
7.2 \\
7.4\end{array}$ & $\begin{array}{l}233.0 \\
23: 5\end{array}$ \\
\hline 634 & 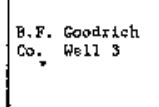 & $645-715$ & " & 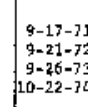 & $\because$ & & $\begin{array}{l}\because: \\
\because \because\end{array}$ & $\ddot{z}$ & $\because=$ & $\because:$ & $\because:$ & & $\begin{array}{l}0 \\
0 \\
0 \\
0 \\
0\end{array}$. & $\because:$ & 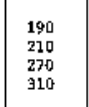 & $\because \because$ & $\because:-$ & $\because:$ & 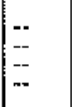 & $\because:$ & $\begin{array}{l}\frac{32}{35} \\
36 \\
47 \\
--\end{array}$ & $\because \because$ & \begin{tabular}{|l|}
2.57 \\
2.56 \\
2.40 \\
2.40
\end{tabular} & $\begin{array}{ll}\because: \\
\because \therefore \\
\end{array}$ & $\begin{array}{l}9,907 \\
1,010 \\
1,200 \\
1,240\end{array}$ & $\begin{array}{l}7.3 \\
7.2 \\
7.3 \\
7.2\end{array}$ & \begin{tabular}{|l}
$25,0.0$ \\
25.0 \\
25.0 \\
25,00
\end{tabular} \\
\hline${ }_{635}$ & 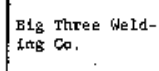 & $639-689$ & " & 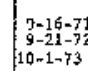 & $\because:$ & $\because$ & $\because:$ & $\because:$ & 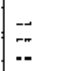 & $\because:$ & $\because$ & $\begin{array}{l}182 \\
1184 \\
106\end{array}$ & $\begin{array}{l}0 \\
0 \\
0 \\
0\end{array}$ & $\because$ & $\begin{array}{l}{ }_{43}^{42} \\
49\end{array}$ & $\because:-$ & $\because:$ & $\Xi$ & $=$ & $\because:$ & $\begin{array}{l}12 \\
\text { 谙 } \\
12 \\
12\end{array}$ & $\because:$ & 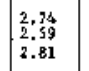 & $\because:-$ & $\begin{array}{l}\frac{4445}{445.1} \\
472\end{array}$ & $\begin{array}{l}7.6 \\
7.2 \\
7.4\end{array}$ & $\because$ \\
\hline .637 & 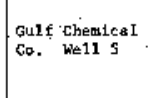 & $630-670$ & s & 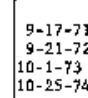 & $\ddot{:-}$ & $\because$ & $\because:$ & $\ddot{\ddot{z}}$ & 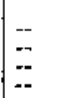 & $\because:$ & $\because$ & & $\begin{array}{l}0 \\
0 \\
0 \\
0 \\
0 \\
0\end{array}$ & $\because$ & 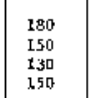 & $\because:-$ & $\because:$ & $\because:$ & $:$ & $\because:-$ & $\begin{array}{l}31 \\
53 \\
22 \\
-1\end{array}$ & 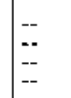 & 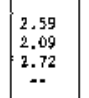 & $\because:$ & \begin{tabular}{l|}
858 \\
7743 \\
7738 \\
779
\end{tabular} & $\begin{array}{l}7.5 \\
7.3 \\
7.5 \\
7.5\end{array}$ & $\begin{array}{l}25.0 \\
25.0 \\
-2.0\end{array}$ \\
\hline${ }^{698}$ & 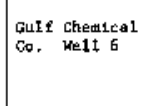 & 634-735 & k & 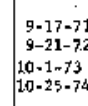 & $\because=$ & $\because$ & $\because$ & $\ddot{\because}$ & $\because$ & $\because:$ & $=$ & & $\begin{array}{l}0 \\
: \\
: \\
0\end{array}$ & 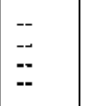 & 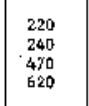 & $\because:-$ & $\because=$ & $\because=$ & $\because:$ & 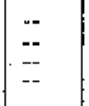 & $\begin{array}{l}40 \\
56 \\
150 \\
--1\end{array}$ & $\because$ & 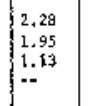 & $\because \equiv$ & $\mid$ & $\begin{array}{l}7,2.2 \\
7,2 \\
7,1 \\
7,1\end{array}$ & $\begin{array}{l}\substack{255.0 \\
25: 0 \\
25: 0 \\
25: 0} \\
\end{array}$ \\
\hline 659 & 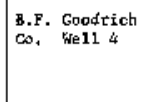 & 620-725 & " & & $\ddot{z}$ & $\because$ & $\because$ & $\ddot{z}$ & $\because:-$ & $\ddot{\because}$ & $=$ & & $\begin{array}{ll}0 \\
\vdots \\
\vdots\end{array}$ & $\ddot{:}$ & $\begin{array}{l}2300 \\
\text { 220 } \\
2200 \\
260\end{array}$ & $\because=$ & $\because$ & $\because:$ & 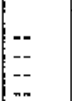 & $\because$ & 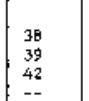 & $\because:-$ & 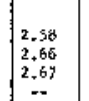 & $=$ & $\begin{array}{l}1,020 \\
1,0270 \\
1,150 \\
1,1100\end{array}$ & $\begin{array}{l}7.4 \\
7,7 \\
7,4\end{array}$ & $\begin{array}{l}255.0 \\
25: 0 \\
25: 0 \\
25: 0\end{array}$ \\
\hline 640 & 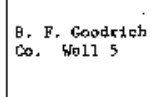 & 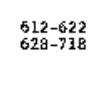 & м & 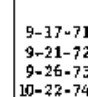 & $\ddot{\because}$ & $=$ & $\because$ & $\ddot{z}$ & $\because=$ & $\because$ & $\because$ & & $\begin{array}{l}0 \\
: \\
:\end{array}$ & $\because$ & $\mid \begin{array}{c}190 \\
190 \\
190 \\
202\end{array}$ & 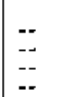 & $\because$ & $\because$ & $\because:$ & $=$ & $\begin{array}{l}25 \\
\text { 35 } \\
28 \\
2--1\end{array}$ & $\because=$ & 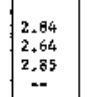 & \begin{tabular}{|l|}
$:$ \\
$\because:$ \\
\end{tabular} & $\left|\begin{array}{c}895 \\
995 \\
9,50 \\
1,010\end{array}\right|$ & $\begin{array}{l}7.4 \\
7, .5 \\
7 ., 5 \\
7.5\end{array}$ & \begin{tabular}{|l}
24.5 \\
as.5. \\
as. \\
25.0
\end{tabular} \\
\hline 642 & Damarar coxpo. & $420-426$ & n & 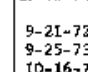 & $\because$ & $\because:$ & $\because$ & $\because$ & $\because$ & $\because$ & $\because$ & $\begin{array}{l}224 \\
220 \\
204\end{array}$ & $\begin{array}{l}0 \\
\vdots \\
0\end{array}$ & $\because:-$ & 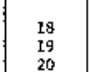 & $\because$ & $\because$ & $\because:$ & 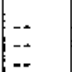 & $\because$ & $\begin{array}{l}18 \\
19 \\
-19\end{array}$ & $\because=$ & 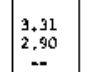 & $\because$ & $\mid \begin{array}{l}419 \\
422 \\
422\end{array}$ & $\begin{array}{l}7.4 \\
8,1 \\
7.6\end{array}$ & $\because:$ \\
\hline 201 & Tho Toxas vo. & 704 & " & 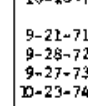 & $\because:$ & $\because:$ & $\because$ & $\ddot{z}$ & $\because$ & $\because$ & $\because:$ & $\begin{array}{l}193 \\
192 \\
194 \\
196\end{array}$ & $\begin{array}{l}0 \\
\vdots \\
\vdots\end{array}$ & $\because:$ & $\begin{array}{l}200 \\
\text { and } \\
\text { and } \\
2000\end{array}$ & $\because=$ & $\ddot{z}$ & $\because$ & 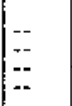 & $\ddot{\Xi}$ & $\begin{array}{l}16 \\
120 \\
20 \\
\cdots\end{array}$ & $\ddot{*}$ & 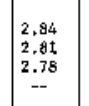 & $\because \because:$ & 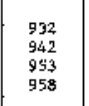 & $\begin{array}{l}7.4 \\
7,4.2 \\
7.5 \\
7.5\end{array}$ & 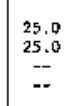 \\
\hline 702 & 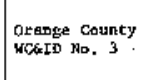 & $6000-672$ & x & 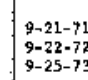 & $\because$ & $\because$ & $\bar{z}$ & $\because$ & $\because$ & $\because:$ & $\because$ & 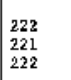 & $:$ & $\because:$ & 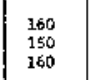 & $\because$ & $\because$ & $\because:$ & $\because$ & $\because$ & $\begin{array}{l}16 \\
\text { 21 } \\
18 \\
18\end{array}$ & $\because=$ & 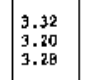 & $\because:$ & 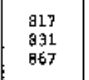 & $\begin{array}{l}7,4,4 \\
7,2.7\end{array}$ & $\begin{array}{l}\begin{array}{c}25.0 \\
25.0 \\
25.0\end{array} \\
25.0\end{array}$ \\
\hline 708 & 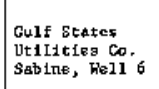 & ${ }_{465}$ & " & & $\because$ & :- & $\because$ & $\ddot{-}$ & $\because$ & $\because=$ & $\because$ & $\frac{261}{279}$ & $:$ & $\because:$ & $\begin{array}{l}50 \\
24\end{array}$ & $\because$ & -: & $\because$ & $\because$ & $=$ & 14 & $\because=$ & 4.00 & $\because:$ & 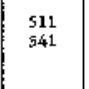 & $7,7.8$ & 23.0 \\
\hline 809 & 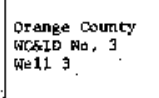 & $570-650$ & is & 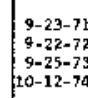 & $\because=$ & $\because \because$ & $\because:$ & $\because:$ & $\because \because$ & $\because:-$ & $\because$ & $\begin{array}{l}236 \\
260 \\
2420 \\
240\end{array}$ & $\begin{array}{l}: \\
\vdots \\
\vdots\end{array}$ & $\because:$ & 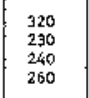 & $\because \because$ & $\because:$ & $\ddot{:}$ & $\because:$ & 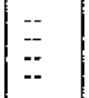 & $\mid$ & $\because \ddot{ }$ & $\begin{array}{l}3.34 \\
3.45 \\
3.47 \\
--.27\end{array}$ & $\because \because$ & 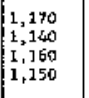 & $\begin{array}{l}7.6 \\
6.9 \\
7.5 \\
7.5\end{array}$ & 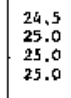 \\
\hline 59-101 & $\mid \begin{array}{c}\text { cityy of oratase } \\
\text { tron }\end{array}$ & $555-666$ & " & 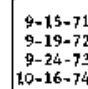 & $\because:$ & $\because:$ & $\because$ & $\because:$ & $\because:$ & $\because:$ & $\because:$ & 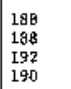 & $\begin{array}{l}0 \\
\vdots \\
\vdots \\
0\end{array}$ & $\because:$ & 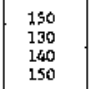 & $\because:$ & $\ddot{\because}$ & $\because:$ & $\ddot{z}$ & $\therefore$ & $\begin{array}{l}50 \\
51 \\
49 \\
4-\end{array}$ & $\because \ddot{0}$ & 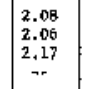 & $\ddot{\because}$ & $\mid \begin{array}{l}751 \\
730 \\
772 \\
766\end{array}$ & $\begin{array}{l}7.3 \\
6.9 \\
8.0 \\
7.2\end{array}$ & $\begin{array}{l}24.0 \\
24.5 \\
244.5 \\
24.0\end{array}$ \\
\hline 103. & \begin{tabular}{|l} 
cetey of orange \\
ber1 2
\end{tabular} & $565-665$ & " & 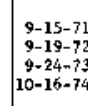 & $\therefore$ & $\because=$ & $\because:$ & $\because:$ & $\because$ & $\because:$ & $\because$ & 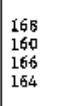 & $\vdots$ & $\because:$ & $\begin{array}{ll}54 \\
45 \\
55 \\
55\end{array}$ & $=$ & $\because:$ & $\because:$ & $\because=$ & $::$ & $\begin{array}{l}35 \\
\text { s. } \\
34 \\
3-\end{array}$ & $\because=$ & 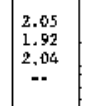 & $\ddot{\because}=$ & \begin{tabular}{|l|}
4620 \\
420 \\
456 \\
446
\end{tabular} & $\begin{array}{l}7.12 \\
6.90 \\
7.2 \\
7.2\end{array}$ & \begin{tabular}{|l}
24.0 \\
ant. \\
24.0 \\
24.0
\end{tabular} \\
\hline
\end{tabular}


Tab1a 3.--Chem1cal analypes of water from vells in the Orange Couaty area, 1971-74-continued

\begin{tabular}{|c|c|c|c|c|c|c|c|c|c|c|c|c|c|c|c|c|c|c|c|c|c|c|c|c|c|c|c|}
\hline $\begin{array}{c}\text { weLL } \\
\text { UJ }\end{array}$ & OMERR & 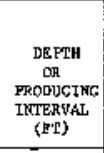 & 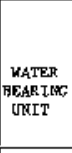 & DATB & 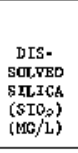 & 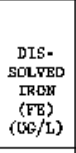 & 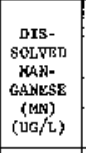 & 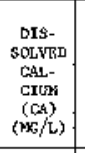 & 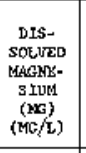 & 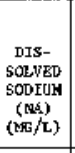 & 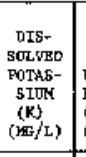 & 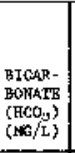 & 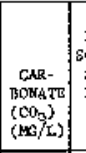 & 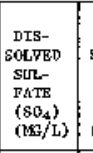 & 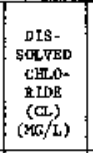 & 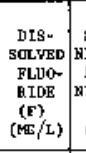 & 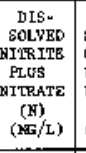 & 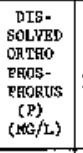 & 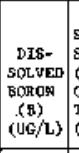 & 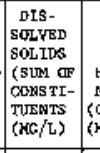 & 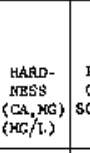 & 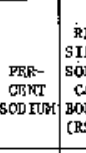 & & 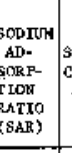 & 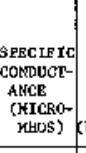 & 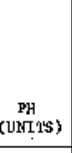 & 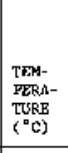 \\
\hline 62-59-104 & $\begin{array}{l}\text { Levingaton } \\
\text { shppyard }\end{array}$ & 749 & M & $\left|\begin{array}{l}9-19-72 \\
9-24-73 \\
1024-74\end{array}\right|$ & $\ddot{z}$ & $\because$ & $\begin{array}{l}-- \\
--\end{array}$ & $=1$ & $\ddot{z}$ & $\because:$ & $\because:-1$ & $\begin{array}{l}211 \\
2008 \\
2014\end{array}$ & \begin{tabular}{|l|} 
\\
$\vdots$
\end{tabular} & $\because \because$ & \begin{tabular}{|l|}
$\begin{array}{l}84 \\
87 \\
78\end{array}$ \\
7
\end{tabular} & $\because \because$ & \begin{tabular}{l|}
-- \\
-
\end{tabular} & $\because$ & \begin{tabular}{|l|} 
\\
-- \\
\end{tabular} & \begin{tabular}{|l|}
$\because$ \\
$\because$
\end{tabular} & \begin{tabular}{l|l}
26 & -7 \\
30 & -
\end{tabular} & $\overline{-}$ & $\begin{array}{l}2.94 \\
2.81 \\
2--1\end{array}$ & $\ddot{z}$ & $\begin{array}{l}623 \\
620 \\
620\end{array}$ & $\begin{array}{l}7.2 \\
7.9 \\
7.4\end{array}$ & $\begin{array}{l}24.0 \\
27.0 \\
27.5\end{array}$ \\
\hline 106 & do & 750 & M & $9-19-72$ & -- & .- & .- & -- & .- & -- & -. & 206 & 0 & n & 86 & -. & .- & -- & -. & -- & 32 & -- & 2.74 & -. & 610 & 7.2 & 25.0 \\
\hline 107 & do & 745 & " & $9-16-11$ & -- & - & -- & -- & $\because$ & - & -- & 216 & 。 & -- & 94 & -- & -. & -- & -. & -- & 25 & -- & 3.04 & -. & 655 & 7.3 & 24.5 \\
\hline 123 & 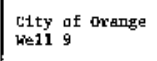 & $529-643$ & " & $\begin{array}{l}9-15-71 \\
9-15-72 \\
\text { and }\end{array}$ & $\because$ & $\because$ & $\because-$ & $\ddot{-}$ & $\because:$ & $\because$ & $\because$ & $\begin{array}{l}204 \\
204 \\
208\end{array}$ & $:$ & $\because$ & $\begin{array}{l}1300 \\
110 \\
120\end{array}$ & $\because$ & $\because$ & $\because-$ & $\because$ & $\ddot{z}$ & $\begin{array}{l}334 \\
36\end{array}$ & $\ddot{z}$ & $\begin{array}{l}2,66 \\
2, .62 \\
2.71\end{array}$ & $\begin{array}{l}-- \\
\because\end{array}$ & $\begin{array}{l}697 \\
706 \\
706\end{array}$ & $\begin{array}{l}7.1 \\
7,3 \\
7,8\end{array}$ & $\begin{array}{l}\begin{array}{l}24.5 \\
25.0 \\
25.0\end{array} \\
25.0\end{array}$ \\
\hline & & & & $\left|\begin{array}{l}9-24.73 \\
10-16-74\end{array}\right|$ & $\because$ & $\because$ & $\because$ & $\because$ & $\because$ & -- & $=$ & $\begin{array}{l}208 \\
208\end{array}$ & $:$ & $\because$ & $\begin{array}{c}120 \\
130\end{array}$ & $\Xi$ & $\because$ & $\because$ & $\because$ & -- & ${ }_{-35}^{35}$ & $\ddot{z}$ & 2.71 & $\ddot{z}$ & $\begin{array}{l}727 \\
737\end{array}$ & $\begin{array}{l}7.8 \\
7.3\end{array}$ & $\begin{array}{l}25.0 \\
24.0\end{array}$ \\
\hline 124 & 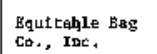 & $590-640$ & м & $\left|\begin{array}{l}9-22-71 \\
9=21-72\end{array}\right|$ & -- & -: & -- & $\ddot{z}$ & $\because$ & $=$ & $\because$ & $\begin{array}{l}179 \\
178\end{array}$ & $:$ & $\because$ & $\begin{array}{l}150 \\
140\end{array}$ & $\because$ & -: & $\because$ & $-\approx$ & -- & $\begin{array}{l}55 \\
55\end{array}$ & $\because$ & $\begin{array}{l}1,83 \\
1.83 \\
1.87\end{array}$ & 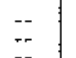 & $\begin{array}{l}741 \\
743\end{array}$ & $\begin{array}{l}7.1 \\
7 \rightarrow 0\end{array}$ & $\begin{array}{l}24.0 \\
25.0\end{array}$ \\
\hline & & & & 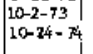 & 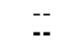 & 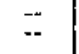 & -- & $\ddot{-}$ & $\because$ & $\because$ & -- & $\begin{array}{l}180 \\
160\end{array}$ & $:$ & $\because$ & $\begin{array}{l}140 \\
140\end{array}$ & $\because$ & $\because$ & $\ddot{--}$ & -- & $\because$ & 34 & $\because$ & 1.87 & $\because$ & $\begin{array}{l}752 \\
769\end{array}$ & $\begin{array}{l}7.3 \\
7.2\end{array}$ & 24,0 \\
\hline 401 & Marathon o11 co & 580 & м & $\mid \begin{array}{l}9.26-73 \\
10-17-74 \\
10-7\end{array}$ & 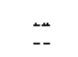 & $\ddot{-}$ & $\because$ & $\because$ & $\because-$ & $\because$ & 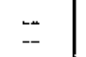 & $\begin{array}{l}176 \\
192\end{array}$ & $:$ & $\because$ & $\begin{array}{l}40 \\
42\end{array}$ & 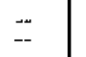 & $\because$ & $\because:$ & $\because:$ & $\because$ & 32 & $\because$ & 2.24 & -- & $\begin{array}{l}422 \\
428\end{array}$ & 7.6 & 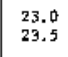 \\
\hline 416 & $\begin{array}{l}\text { Leyingston } \\
\text { slippyard }\end{array}$ & $650-730$ & M & $|10-24-74|$ & $-\cdot$ & -- & -. & - & -- & -- & -- & 195 & 0 & -- & 190 & -- & -- & -- & -- & $\therefore$ & -. & $\cdots$ & -- & $\cdots$ & 902 & 7.4 & -- \\
\hline & & & & & & & & & & & & & & & & & & & & & & & & & & & \\
\hline & & & & & & & & & & & & & & & & & & & & & & & & & & & \\
\hline & & & & & & & & & & & & & & & & & & & & & & & & & & & \\
\hline & & & & & & & & & & & & & & & & & & & & & & & & & & & \\
\hline & & & & & & & & & 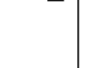 & & & & & & & & & & & & & & & & & & \\
\hline & & & & & & & & & & & & & & & & & & & & & & & & & & & \\
\hline & & & & & & & & & & & & & & & & & & & & & & & & & & & \\
\hline & & & & & & & & & & & & & & & & & & & & & & & & & & & \\
\hline & & & & & & & & & & & & & & & & & & & & & & & & & & & \\
\hline & & & & & & & & & & & & & & & & & & & & & & & & & & & \\
\hline & & & & & & & & & & & & & & & & & & & & & & & & & & & \\
\hline & & & & & & & & & & & & & & & & & & & & & & & & & & & \\
\hline
\end{tabular}




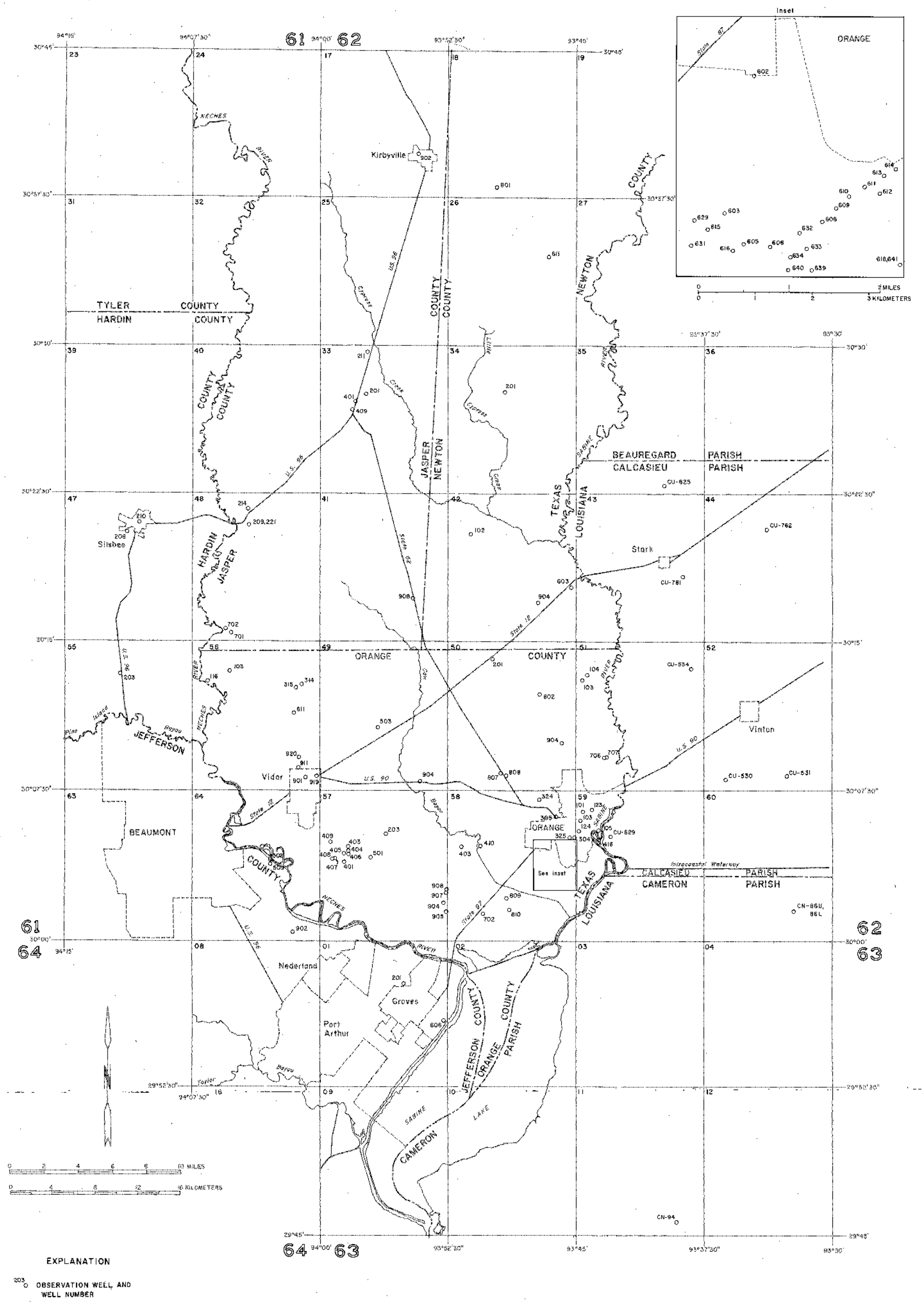

Figure 2 


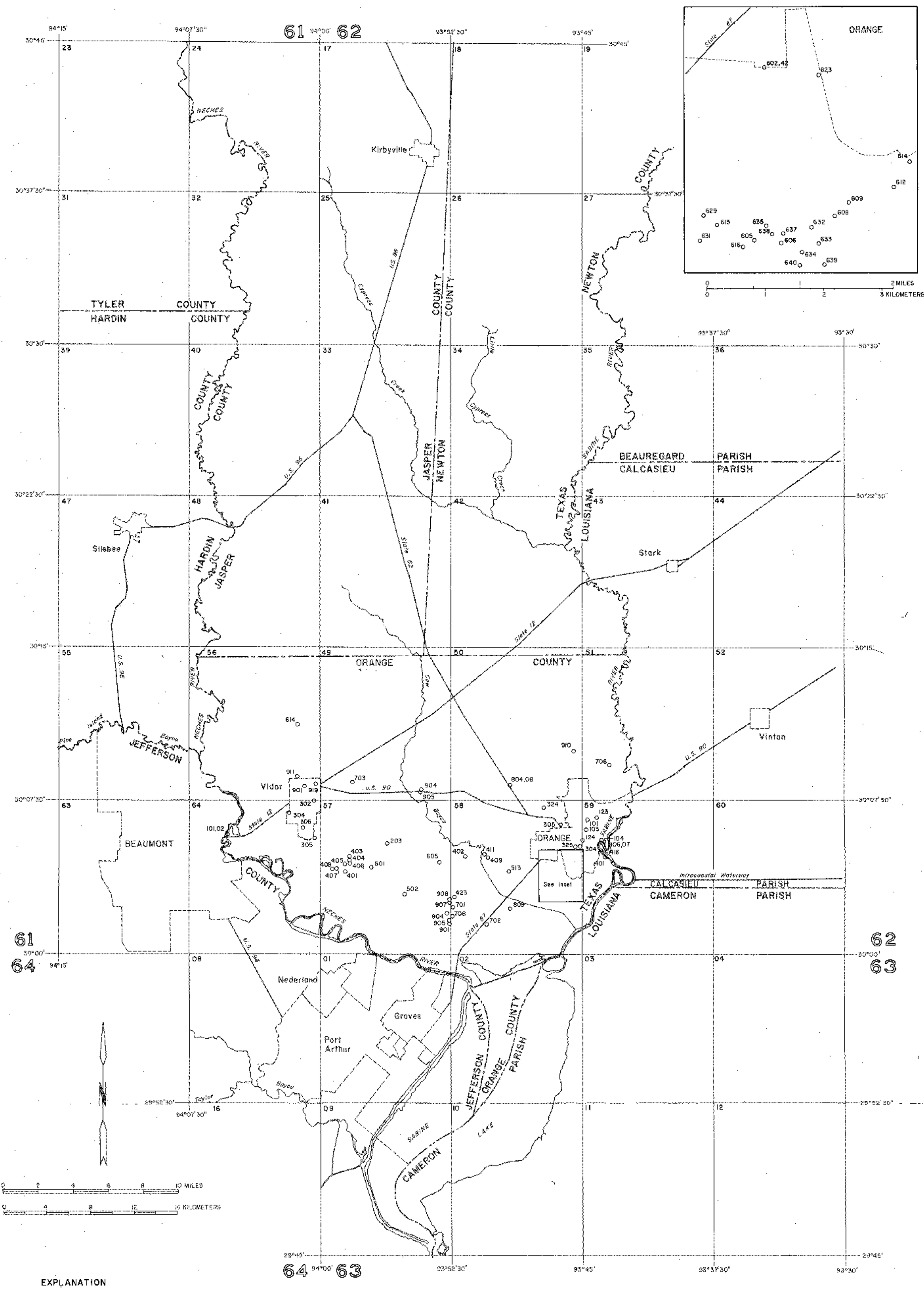

203. OQSERVATION WELL AND
WELL NUMBER 

